\title{
Beiträge zur rom. Lant- und Formenlehre.
}

Das Studium der historischen Grammatik der einzelnen romanischen Sprachen erfreut sich im letzten Decennium ungemein reger Teilnahme: alte Sprachdenkmäler werden sorgfältig in Laut- und Formenlehre untersucht; den noch lebenden Dialekten wird immer mehr Aufmerksamkeit geschenkt; Monographieen in Form von Zeitschriftenaufsätzen oder Doktordissertationen suchen über alle möglichen Punkte genaueste Auskunft zu geben. Es dürfte daher nicht mehr verfrüht sein, wieder zur vergleichenden Methode zurückzukehren und dabei namentlich schärfer als es bisher der Fall, zu scheiden, was gemeinromanisch ist, was sich nur aus der Sonderentwickelung der einzelnen Idiome erkärt; ferner die Reihenfolge der einzelnen Lautgesetze zu bestimmen. Nur so wird es möglich sein, die ethnologischen Fragen sicher zu lösen, mit denen man sich neuerdings, vielleicht etwas $z u$ frühe, wieder häufiger beschäftigt hat; nur so kann man oinen Einblick in die Geschichte des Volkslateins bekommen, in die Fortentwickelung des Lateinischen im ersten Jahrtausend unserer Zeitrechnung, worüber uns die lateinische Sprachwissenschaft nur sehr spärliche Auskunft giebt. Die Abweichungen vom klassischen Typus sind darin von doppelter Art. Die klassische Form weicht von der nach Malsgabe der lateinischen Lautgesetze zu erwartenden ab, die vom Consensus der romanischen Sprachen gegebene stimmt mit jener hypothetischen überein: in diesem Falle ist es klar, dafs der Romanist sich nicht weiter um die Sache zu kümmern hat: aus ital. ebbi sic. ibbi läfst sich auf ein * hébui schliefsen, das aus *hebi entstanden ist, wie * venuui aus vẽni, *vĩdui aus vidi u. a., * häbi selbst ist das regelmälsige starke Perfekt zu habeo wie feci zu facio, wie jeci zu jaceo; im Osk. ist es erhalten. Ist aber die Sache umgekehrt, so darf sich der Romanist nicht, wie es gewöhnlich geschieht, mit der Aufstellung eines ihm passenden Typus begnügen, wird vielmehr den unrechtmärsigen Sprölsling, den er geschaffen hat, auch legitimieren müssen; thut er es nicht, so hat er die Sache nicht erklärt. Ital. nuora span. nuera cat. prov. nora sic. nora rum. noră spätlat. nora Marini Pap. Dipl. LXXVI (Paris 7. Jahrh.) einerseits, lat. nūrus skr. snusha gr. vvós sl. snucha alb. snuse deutsch schnur, die alle auf einen Typus mit $\vec{u}$, allenfalls $\ddot{u}$, zurückgehen, andererseits lassen sich lautlich nicht vereinigen, der Indogermanist wird die lateinische Form ganz in der Ordnung 
finden, und das späte lat. nora als blofsen Schreibfehler betrachten, da $\partial$ oder $\bar{o}$, soviel wir bis jetzt wissen, in keinem Ablautverhältnis zu $\bar{u} \quad \bar{u}$ steht; wenn nun der Romanist nachweist, dafs im lat. wirklich nora existierte, so wird er zugleich auch darauf hinweisen, dafs diese Form an Stelle der regulären getreten ist durch Einflufs von söcra; denn zwei Worte, die in einer engen ideellen Beziehung zu einander stehen, beeinflussen sich häufig, zu den von anderen schon genannten füge ich hinzu sic. jornu nach notti. ${ }^{1}$ - Um nun freilich unterscheiden zu können, was jung und was alt ist, wo das klassische Latein, wo das Vulgäre oder das Gemeinromanische? regelmälsig (sit venia verbo) sind, ist eine genaue Kenntnis der lateinischen Lautgesetze nötig; ein Erfordernis, dem gegenwärtig um so weniger leicht Genüge geleistet werden kann, weil das am meisten citierte Werk, Corssens Vokalismus heute noch viel weniger als beim Erscheinen der zweiten Auflage dem Standpunkte der Wissenschaft entspricht und eigentlich nur noch den Wert einer (häufig recht unglücklich geordneten) Materialsammlung hat. Ich gedenke nun im folgenden eine Reihe Fragen der romanischen Grammatik historisch-vergleichend $\mathrm{zu}$ untersuchen: nicht in bestimmter Auswahl sondern wie sie sich mir gerade bieten. Einleitend stelle ich jedesmal das betreffende Kapitel der lat. Grammatik dar; nicht mit dem ganzen Apparate der vergleichenden Grammatik und nicht erschöpfend, sondern mehr summarisch, so viel eben für das Verständnis im betreffenden Falle nötig ist.

\section{Die Behandlung tonloser Paenultima.}

Jeder nachtonige, nicht in letzter Silbe (es ist dabei vorausgesetzt, dafs $-i$ der Personalendungen schon gefallen sei) stehende Vokal wird im Lateinischen zu $e$ geschwächt, dieses $e$ bleibt vor $r$, vor mehrfacher Konsonanz, nach $i$; vor $l$ wird es zunächst $o$, das nach $e i$ stets, nach $v$ bis zur Zeit des Augustus bleibt, in den übrigen Fällen und nach $v$ seit Augustus zu $u$ wird: vor $p b f m$ tritt teils $u$ teils $i$, vor den anderen einfachen Konsonanten $i$ ein. Die Verhältnisse vor $v$ sind äufserst verwickelt, kommen aber aufser in einem gleich zunennenden Falle für uns gar nicht in Betracht, ich übergehe sie also. - Dieses Gesetz ist älter als die klassische jünger, wie es scheint, als die indogermanische Betonung, da aber für das Romanische durchaus die erstere malsgebend ist (die scheinbaren Ausnahmen kommen unten zur Sprache), so nehme ich auf die vor Eintreten des daktylischen Accentsystems nachtonigen, nachher aber betonten Silben keine Rücksicht. Unter nachtonig verstehe ich im folgenden der Kürze halber nur die tonlose Paenultima, nie die letzte Silbe.

1 Nicht anders neap. pimece (cimex) wegen purcce; oder Ghelf wegen Ghibellini in den Chron. Per.

2 Vulgärlat. nenne ich die thatsächlich überlieferten, gemeinrom. die durch mehrere rom. Sprachen erwiesenen Formen.

3 Vergl. L. Meyer Bezzenbergers Beiträge I $143 \mathrm{ff}$. 
Die Beispiele zu dem genannten Gesetze werden sich im Laufe der Arbeit finden, sie hier anzuführen, scheint mir unnötig, es mag genügen, wenn ich die Ausnahmen aufzähle und erkläre.

$a$ steht in Nachtonsilbe zunächst in spätaufgenommenen Fremdwörtern; solche, die vor oder während der Wirkung des Gesetzes in die Sprache eintraten, unterlagen ihm, wie z. B. machina trutina. So haben wir hilaris stomachus monachus hebdomada cithara pelagus, namentlich wenn der Tonvokal schon $a$ ist: lampada sabbata calamus mataris samara. In letzterem Falle erscheint $a$ auch in echt lat. Wörtern (Assimilation), doch, so viel ich sehe, nur wenn der Schlufskonsonant der Tonsilbe eine Lipuida ist: alacer cannabis, anatem, dagegen pampinus lapidem. Verschieden ist farfarus, wo nicht der Tonvokal allein, sondern die völlige Gleichheit der beiden Silben die Schwächung hinderte. In atavus abavus concavus hat das Bewufstsein der Zusammensetzung die Schwächung des $a$ aufgehalten. In Caesar- jubar- liegt Einflufs des Nominativs vor. Versuche, auch in diesen Fällen z. T. die regelmälsigen Formen durchzuführen, fehlen nicht: das rein physiologische Gesetz der Schwächung, das physiologisch-psychologische der Assimilation, das psychologische des Einflusses des Nom. auf die übrigen Formen, des einfachen Wortes auf die Zusammensetzungen kreuzen sich: hier siegt das eine, dort das andere. So lesen wir monicus (Schuch., Vok. III 101), anites (Lachmann zu Lucrez S. 16, Schuchardt I 197) farferi (Plaut. Schuchardt I 196)' ${ }^{1}$ materis (ib.) samera (ib.) Caeseris (Schuchardt I 195). Ob in den Fällen vor $r$ nicht vielmehr jenes toskanische Gesetz vorliegt, nach welchem tonloses $a$ vor $r z \mathrm{zu} e$ wird, ist nicht auszumachen. - Umgekehrt steht $a$ gegenüber klassisch $c$ in camara carcares sisarum, deren erstes und drittes griechisch sind, ob das zweite, ist fraglich. $\mathrm{Da}$ kein got. Lautgesetz existiert, wonach aus * karkera karkara entsteht, so muls entweder auf bestimmtem lat. Gebiete die Assimilation des zweiten Vokals an den ersten (zu erklären wie in farfarus) älter sein als die Assibilation (die rom. Sprachen weisen alle ohne Ausnahme auf assibiliertes $c$, wo also $a$ in der Endung erscheint, ist es jung und anders zu erklären als im Lat.) oder das got. Wort ist nicht aus dem Lat., sondern yus dem Griechischen, wo aus carcer xápxapov entstehen konnte, entlehnt; kymr. carcharawr kann fürs Lat. nichts heweisen.

$e$ findet sich, aufser vor $r$ und nach $i$, wo es regelmälsig ist, durch Assimilation in vegetus (besser gesagt, das $e$ der Tonsilbe schützte das $e$ der folgenden tonlosen vor Übergang in $i$ ) hebet-, seget- teget-, wo auch der Nominativ mitwirken konnte (aber equitpedit- wurden durch mülit- bestimmt); in Zusammensetzungen, wo das Primitiv Einflufs übte: impetus duodecim. Unerklärt ist juevenis. $i$ steht vor $l$, wenn im Auslaute $i$ steht, so erklären sich die Adj. auf -ilis, neben denen noch die alten endungslosen Neutra

1 Auch farfenum Paul. Diac. 88, 13 ist wohl in farferum, nicht farfarum zu bessern. 
als Adv. vorkommen: semol, später * semul 1 facul. Die Formen facile simile sind Neubildungen (unrichtig ist, was Studemund bei Horning, Lateinisches C S. 26 darüber lehrt). Vor Labialen hat $i$ einen zwischen $i$ und $u$ liegenden Klang, den Kaiser Claudius durch ein besonderes Zeichen ausdrücken wollte; vgl. Schuchardt II 197 ff., $218 \mathrm{ff}$; wo Grammatikerzeugnisse und Belege zur Genüge gesammelt sind. Für unsern Fall reichen folgende Bemerkungen aus: vor $m$ ist der Übergang zu $i$ ganz durchgeführt, die Erklärung von franz. -ons aus ùmus, obschon von Rothenberg, Herrigs Archiv $62,160 \mathrm{ff}$. wieder aufgefrischt, findet heute wohl keine Anhänger mehr; was Caix, Vocal. Ital. 14 aus dem Chianaj. beibringt, ist falsch: der Vokal der Nachtonsilbe wird stets durch denjenigen der auslautenden bedingt. ${ }^{2}$ Vor $p$ erscheint $u$ im Lat. stets in occupo, neben $i$ in dissupo, das man, kaum mit Recht, dem ital. sciupo zu grunde legt. Mit $f$ haben wir nur aurifex neben aurufex, die rom. Formen fordern das erstere.

o steht, aufser in eolus iolus, vor $\mathbf{r}$ in einem Teile der neutralen s- und $r$-Stämme und in Adj. sowie in arbor. Nur in marmor- memor- ist das $r$ alt; ob das $o$ vom Nominativ eingeschleppt oder vor $r$ nicht geschwächt worden ist, läfst sich nicht entscheiden, mit Rücksicht auf das folgende werden wir ersteres für wahrscheinlicher halten. Sicher stammt das 0 in tempor-aus dem Nom., die alten Formen bewahrt gener- vgl. gr. yéveos sl. slovese air. tige; was Corssen II I 69 ff., Caix Voc. It. 8 f. sagt, ist falsch.

u: fulgur- guttur- murmur- sulfur- satur- cicur-. Überall scheint das $r$ alt zu sein, sehen wir von murmur- ab, das nichts beweisen kann, da das zweite $u$ vielleicht entstanden ist, wie das $a$ in farfarus, so bleiben drei Beispiele mit $u$ in der Tonsilbe, doch ist Assimilation bei folgender Doppelkonsonanz nicht wahrscheinlich; vielmehr wird in allen Fällen das $u$ vor altem $r$ aus $\delta$ entstanden sein; der Nomin. hatte $\partial r=$ idg. $r$, memor dagegen hatte einst $\bar{o}$ im Nominativ, daher die Differenz.

Ich muls hier einige Fehler des lat. Lexikons verbessern. Klotz setzt an: panicum burricus carpinus. Für keines werden Dichterstellen gebracht; die Angabe der Quantität beruht lediglich auf Analogieen oder Etymologieen. Die rom. Sprachen verlangen panicum Ascoli, Arch. IV 353 Anm. 3, burricus it. bricco neap. borrico nprov. bourico comsk. borik u. s. w., cárpinus it. carpine sp. pg. carpe frz. charme rum. cárpin.

Unter gewissen Bedingungen ist endlich im Lateinischen synkope eingetreten, unter welchen, ist schwer festzustellen; was Corssen in seinen verschiedenen Werken und E. Martini, Riv. di fil. class. VII

1 Die klassische Form sïmul lehnt sich an simïlis an; die organische sềmol ist im Romanischen erhalten.

2 Alle von Caix zur Stützung seiner These aus dem Chianaj. angeführten Beispiele beweisen gar nichts, weil überall (wenigstens in Billis Poesie) dieselbe Assimilation vorliegt, ich werde daher im folgenden nie auf sie Rücksicht nehmen. 
darüber sagt, ist heute völlig wertlos. Mafsgebend sind die umgebenden Konsonanten und der Accent. Dabei scheint ein umspringendes (also wohl das indogermanische) Betonungsgesetz zu wirken, auch in Fällen, die verhältnismä/sig spät entstanden sind, ardor z. B. wird von arždus = asïdus nicht getrennt werden dürfen, das $i$ kann aber erst nach Eintreten des Rhotacismus gefallen sein, da aus *asdōs a ados entsteht; dasselbe ist zu bemerken bei urtica, wenn es zu urere gehört (usitica uritica). Ich hoffe an anderm Orte die Sache ausführlich darlegen zu können, und mache hier nur auf folgende Paare aufmerksam: validus valde, avidus audere, calidus caldus, frīgidus frìgdus, solìdus soldus, larìdum lardum, viridis vĭrdis, *novidus nūdus, *cruvidus crūdus, uvidus üdus; zu diesen lateinischen Beispielen kommt noch ein gemeinrom. lūrìdus lürdus, $\mathrm{zu}$ erschliefsen aus it. lordo sp. lerdo frz. lourd neap. lurdē; aus lurždus hätte in letzterer Sprache nur *lurětē entstehen können, lurdē weist auf synkopierte Vorlage hin; lat. $\tilde{u}$ rom. $O$ kommt dabei nicht in Betracht $\mathrm{Ob}$ matlus $={ }^{*}$ maddus aus madidus sei, ist zweifelhaft, dals $d d$ zu $t t$ werden kann, ist möglich. - Wenn meine Annahme richtig ist, dals ursprünglich fríg gùdus aber z. B. frigg $d^{\prime}$ ( $-e$ in Endungen, sofern es alt ist, trug stets den Accent) gesagt wurde, so erklärt sich auch der Vokal des rom. frẹddo: das Vorrücken des Accents um eine Silbe hat Verkürzung des dadurch tonlos werdenden Vokals zur Folge. Förster glaubt, frīgdus in rum. frig sp. frio sehen zu dürfen Rhein. Mus. XXXIIl 297; mit Unrecht, rum. frig ist das zum Adj. gewordene Subst., ein Vorgang, der weit häufiger ist, als Diez glaubte, sp. frio kann ebenso entstanden sein, oder $-\check{z} y$ - wurde zu $i$ auf lautlichem Wege, vgl. stria $=$ striga.

Als lateinisches Beispiel der Synkope erwähne ich noch auca aus avica, wie audere aus av(i)dere, dagegen ist nauta nicht aus nivila entstanden, sondern das gr. vavi $\tau \eta$, ein dem griechischen $-\tau \eta / S$ entsprechendes lat. Suffix -ta existiert nicht.

Nach diesen Vorbemerkungen durchgehe ich die einzelnen romanischen Sprachen. Es war mein Bestreben, möglichst alle Fälle zu sammeln, ich hoffe wenigstens nichts Wichtiges übergangen zu haben. Die Behandlung von $\leq c \in l \in$ habe ich absichtlich weggelassen, die Fragen, die sich daran knüpfen, fordern sehr eingehende historische Studien und ein Material, über das ich noch nicht verfüge. Die Beispiele sind nach den Schlufskonsonanten der Nachtonsilbe geordnet: $c t p, g d b, n m r s$. Verba, bei denen stets Ausgleichungen zwischen stamm- und endungsbetonten Formen haben stattfinden können, führe ich nur an, wo sie von besonderer Wichtigkeit sind.

I. Rumänisch purece ferece foarfeci soarice und soaricu"

1 Der Anlaut ist auffällig ,mit parasitischem i“ Miklosich, Lautl. IV 79 ist keine Erklärung. Hat $\mathscr{\varrho} \varrho \alpha \xi$ Einflufs geübt? Oder assimilierte sich der Anlaut dem Inlaut? Vgl. S.212.

Zoitsohr. f. rom. Ph. VIII. 
păntece vitreg mantżă piedică bisirica; - deget cumpet preamet gemet u. a. Verbalabstrakta auf $-t$, cucurbeta, wenn es volkstümlich ist; neted muced fraged marced rapped putred treaped umed vilced veşted ${ }^{1}$ lanced sarbed ${ }^{2}$; limpede geht auf ein, durch Assimilation entstandenes limpidis (vgl. viridis) zurück; zum Teil treten Formen mit $t$ statt $d$ auf, wie in den süditalienischen Dialekten, Diez verzeichnet umet, Miklosich, Lautl. IV 14 fraget mrum. lângetu; - arpin frasin noatin galbăn gemin paltin (Suffixangleichung an carpin frasin) peapen pecingine funingine. Unbekannt ist das Etymon von curpen; Pl. auf -uri (die Inf. auf -ere in subst. Funktion beweisen nichts, wenn meine lat. Neutr. I 4 I f. gegebene Erklärung richtig ist), fulger uger murmure u. a. - paghina erweist sich durch $g h$ als Lehnwort aus dem Griechischen; femeiä kann nicht femina, allenfalls * feninia sein, besser familia nach Miklosich a. a. O. III 39. In culég (cóllǐgo) hat die Betonung des Infinitifs den Sieg davon getragen, sparángă scheint zunächst aus Griechenland zu kommen.

Synkope erscheint in den lat. Fällen cald verde. Dann in doamne dominus, schon vulgärlat. (vgl. Schuchardt II 4 I I; Chitsu Col. luĩ Trai. Neue Serie III Heft 6; die Indices zum C. I. L). Man könnte darin einen Fall der Verkürzung eines sich an den folgenden Eigennamen anlehnenden Titelwortes sehen (vgl. frz. sire ital. sor sora u. a.). Allein da domnus schon plautinisch ist, so liegt es näher, die Synkope für alt, aus abstufender Deklination zu erklären, also dóminus domní, oder ursprünglich dubenos domni (wobei die alte Etymologie = skr. damana aufzugeben ist). In ermu éremus haben sich die beiden Liquiden angezogen. Auffällig ist salce salca, alb. selk; weshalb fällt hier $\check{i}$ und weshalb geht $l$ nicht in $r$ über? In detoriu und imbet ist zunächst das $b$ ausgefallen, dann der tonlose Vokal mit dem betonten verschmolzen. scoarfa ist nicht cortic-, wie Cihac sagt, sondern scortea Diez, Wb. +288 ; Miklosich, a. a. O. III 7 I.

Was endlich die Qualität des tonlosen Vokals betrifft, so haben wir vor $n$ stets $i$, vor den übrigen Konsonanten teils $i$ teils $e$, ersteres moldauisch; dafs im Urrumänischen nur $e$ galt, zeigt der gänzliche Mangel der Assibilation. In galbăn liegt Assimilation vor. Vor $r$ erscheint zuweilen $u$ (lature iedura virgurä) unter Einflufs der PI. auf -uri und der Formen iepure marmurat.

2. Sicilianisch (nach Biundi und Mortillaro) cimicia cortici filici forfici ilici larici pumicia salaciu silici - tralciu (Italianismus?) purci surci - panza. pillicu (Caix, Studi 45I) musicu monicu reficu carrica (aber carcári) die Verba auf -iare = icare oder idjare vasca frasca nasca chianca cosca; jiditu (aus dijitu) gavila guvitu limitu und zahlreiche Verbalabstrakta auf żla von $a$-Verben; Synkope nur in crettu (scriatu) sofern es.von crepitus stammt, was nicht ganz

1 Cihac leitet das erste der zwei Worte von *livicidus, das zweite von viescidus (aus viescere) ab: beides unmöglich. Ich führe beides auf viscidus (vgl. später) zurück, im erstern sehe ich eingeschobenes $l$.

* Nicht exsupidus, wie Cihac will, sondern exalbidus. 
sicher ist, und in dettu, dessen $e$ statt $i$ nach der Toskana weist; - spirdu purpu (polypus). - Dic Adj. auf -zdus werden verschieden behandelt: acitu stolitu nsipitu rancilu sfravitu sfuncitu und sfincilu valitu viscitu scomitu trispitu. selbst amitu. 1 Daneben fradiciu, wovon später, limpiu nfuciu marciu musciu; sciarvidu; rattu; nellu ist wegen $e$, riggidu wegen $g g$ Italianismus. Die gemeinrom. caudu friddu virdi sodu, neben letzterem in gleicher Bedeutung sozzu (also nicht ital. sọiżo!) sol(z)dius. ${ }^{2}$ - bustu. - purpaina furraina und furrania pilinia ncunia (gleichsam *incugine $(m)+a$, das seltene -udine $(m)$ wurde fast überall durch das häufigere ugine $(m)$ ersetzt), pirainu, filina. Daneben kennt das Sicil. ein Suffix aggini, das wie das ital. aggine Abstrakta von Adjektiven bildet; auch andere Dialekte kennen einen doppelten Typus: agine $(m)$ in populärer Behandlung in den alten Wörtern, -aggine in den Neubildungen; nur im Toskanischen ist der Unterschied kaum merklich, woraus folgt, dals aggine in den Dialekten aus der Schriftsprache stammt. In den angeführten Fällen geht aina ania (vgl. addaniu = daino) auf agine $(m)$ nicht auf agne(m) zurück, vgl. sfuiri lejiri rijtuni, das schon genannte jiditu u. a. fimina carpinu und carpanu. Daneben donna und lanna, beide schon lateinisch. - Ich erwähne hier, dafs im Nexus $s m$ stets $i$ eingeschoben wird risima fantasima fanatisimu incantisimu cugghiunisimu, biasimu ist Lehnwort, echt ist gastimári, also keine Synkope. - carzari marmaru cancaru u. a.; Inf. auf -iri, Pl. auf -ura inasu.

Im allgemeinen unterbleibt die Synkope auch hier, doch ist sie bei anlautend $r$ und auslautend $c$, so wie bei anlautend $l$ auslautend $p$ beliebt. Bemerkenswert ist, dals die Fem. auf -ca synkopieren, die Mask. nicht. Der Vokal ist meist $i, a$ erscheint, wo die 'Tonsilbe $a$ enthält; zu den schon gebrachten Beispielen vgl. atamu astracu (lastrico) calaciu ansara annata saraco (sargus) sparaciu, ferner smimmaru (aber, aufser den Infinitiven, z. B. soggira wegen des Palatals). 3

3. Neapolitanisch (eine Untersuchung über das Neap. wäre von grölster Wichtigkeit, die Dialektwörterbücher sind hier bei weitem nicht so zahlreich und reichhaltig wie für andere Dialekte; Puoti z. B. bietet verhältnismälsig wenig; auch der alte Galiani läfst manches $\mathrm{zu}$ wünschen übrig. Mögen also Leute, die an der Quelle sitzen, hier und im folgenden meine Arbeit ergănzen). surece und soraca pimece (cimex vgl. S. 206) polece pommece ilece ermece (imbrex) filece, nudeco riseco puonteco muozzeco und andere Bildungen auf ico; ghiedeta (Umstellung wie im Sicil., daneben rito aus der Schriftsprache) pideto und andere Abstrakta, guveto tiermete chicjelo prevete; muceto fraceto acito gravito grancito commeto culi-luceto fuceto

1 Vgl. noch mpatiddiri aus * mpadditiri wie mpulisari aus imbossolare.

2 linnu $=$ ital. lindo nicht limpidus.

3 Ich bemerke ein für allemal, dafs ich bei den Dialekten nur das Wcsentlichste anführe, und Übereinstimmungen mit der Schriftsprache, wo sie nicht von Wichtigkeit sind, übergehe. 
cupeto propeto (= proprio, propile auch in Accumoli Abbruzz. Ult. II Papanti S. 62) liqueto spruceto lapete treppete pisita amito. Daneben lurdo (S. 209) cavodo aus caudo ', ferner suzzo und tiepolo wovon später.' - femmena lentineja petineja folinneja propajena ancuneja cesiuneja ${ }^{3}$ (*testugo statt tesiudo). Die Formen decken sich mit den entsprechenden Sicilianischen, nur ist die Umstellung des $i$ im Neap. konsequent durchgeführt, aus ia mufste, da das Neap. den Hiatus nicht duldet, eja entstehen, vgl. capitanejo (capilanius statt -nus, wie in den Chroniken von Aquileja und Perugia, Hist. Rom., prov. u. s.) caterveja u. a. - sm wird behandelt wie im Sicil.: resima antosecaseno (sic!).

4. Tarentinisch (nach De Vincentiis und Morosi Arch. Gl. IV I 7 ff., wo der Sache nicht die gehörige Aufmerksamkeit geschenkt ist). podice manice romice aber sciorgio (sorex, wegen des Anlauts vgl. S. 209 Anm. 1; Lecce surgi 1 40) curcu nasca naca selbst ušco und oscra; discito cuscito javito pidito vuvito preite criettu (Lecce); vurpo (Lecce purpu). mucilu fungitu cavilo, truvolo aber Lecce trubbu mit Verlust der Endung; fiezzo ist nicht foetidus, es könnte *foetius sein Flechia Riv. fil. cl. II 191, dagegen Ascoli Arch. IV I 25 Anm. 2; besser palst fótidius, woraus fetitito fiezzo, die Synkope ist in diesem Ausnahmefall zu erklären wie in ital. netto, wovon hernach. ncutina piluscina pitiscina prubascino"; in Lecce aber felinia (140), entweder weicht der Dialekt von Lecce hier vom Tarentinischen ab, oder die Form ist auf irgend eine Art aus dem Neapolitanischen eingedrungen; merke ponnula aus polline 139 . Bei $r$ unterbleibt die Synkope, der Vokal ist teils $i$, so in den Inf., in den Pluralen auf $r$, und sonst; $a$ erscheint vor $r$ wie auch vor anderen Konsonanten, wenn'die Tonsilbe $a$ enthält, tar. pastano (neben pàssili und dem schon genannten javilo) häufig in Lecce S. I37. 139.

5. Sardisch. Man spricht wohl nicht selten von einer gewissen engern Verwandtschaft des Sardischen mit dem Spanischen; sehen wir von dem rein zufälligen längern Bestehen des auslautenclen $-s a b$, worin alle nördlich der Apenimen gelegenen romanischen Idiome im Gegensatz zu denjenigen der italienischen Halbinsel im engsten Sinne, der südlichen Inseln und Rumäniens bis auf einen gewissen Grad übereinstimmen, so lälst sich kaum etwas schlagendes anführen, in manchen Dingen, so namentlich in der Behandlung

1 Vgl. avoto favozo fravoto savotamvanco mavotone, welch letzteres die Erklärung von ital. mattone aus maltha als allein richtig erweist, vgl. Arch. Gl. IV 474. Baist hätte dies Ztschr. V 562 bcrücksichtigen und sich und andern eine Erklärung ersparen können, die er jetzt Rom. Forsch. I 443 widerruft.

2 Wenn daneben muscio (lento fiacco) vorkommt, so handelt es sich nicht um Ausfall des 늘, was hier unerhört wäre, sondern um eine Rückbildung ans musceto.

${ }^{3}$ Zum Anlaut vgl. cellecare = titillicare (Arch. II 319) und cienero (tener).

- Das Wort ist masc., also streng genommen nicht lat. propago sondern ein von propaginare gewonnenes propaginum. 
tonloser Paenultima, trennt sich das Sard. durchaus vom Span. Ich lege das logudoresische (nach Spano) zu grunde.

codighe poddighe pulighe filighe ilighe und elighe salighe sorighe dentighe seneghe' pesadighe maniga tuniga bertiga persighe meigu; nadia fidiu; mit Suffixvertauschung léttiga bedrigu; pancia cagl. mancia (mantice) - ispau it. spago von Caix Studi 53 aus sparticum erklärt lautlich unmöglich, eregiu ist nach sard. Lautgesetzen kaum zu erklären, es dürfte wie so viele sard. Wörter aus dem Span. stammen, monzu geht auf * monicus monichus zurück, griech. $\chi$ verstummte rascher

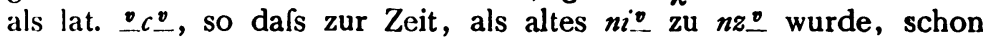
eine Form *monius bestand. didu (nicht *digtum sondern di(g)itum) cuidu boidu coidu piaitu comite fomili limite semida; maladiu (aus malaidu, wie das Wort im Cagl. wirklich lautet, vgl. noch log. parua aus paura); auch hier sind die Abstrakta und Part. auf ztus von $a$-Verben häufig, duda ist wieder ein span. Lehnwort. - colpu polipu abile aus alibe adzbe adeps. - isparau - luzzidu und luzzigu log. lucitu; gall. rigidu bischidu candidu fulgidu fungidu ranchidu suzzidu frazigu tribidi labida raidu - limpiu tepiu marciu (netta stammt aus dem Ital., wie e zeigt); die lat. birde fridu (cagl.) frittu (log.) caldu; endlich eine Anzahl Neubildungen: aspidu (aspro insipido) pidigu (nero aus *pighidu zu pix) u. a. - cannau - probaina sastaina tostoina ferraina imbena cisini pampinu nebst zahlreichen Adj. auf -inu: múrino (nero) muirtinu (rosso) ispidinu (magro) chidrinu u. a., ferner cagl. rexini radix aus * radicem ${ }^{*}$ racidem ra(c)inem, oder eher aus ra(d)icinem; gall. trebini weist auf eine Flexion *trepes *trepinis vgl. pg. trempe und Ascoli, Arch. IV 398 ff., mein Neutr. 70.

Es ist schwer in diesen verschiedenen Formen, namentlich bei den Adj. auf zdus, ein einheitliches Gesetz zu finden, verschiedene Dialekte kreuzen sich offenbar, im Log. sollte $g^{\prime}$ vor $i$ guttural werden, fulgidu kann aus der Schriftsprache stammen, aber fungidu? Nicht weniger auffallend ist die Behandlung von -cid-. Auch polipu neben colpu, das $p$ des erstern lăsst an der Volkstümlichkeit des Wortes zweifeln. - Der tonlose Vokal ist gewöhnlich $i$, auch in Fällen, wo das lat. $a$ hat; nur vor $r$ schwanken die Dialekte, Cagl. zieht auch hier $i$ vor, während Log. häufig $a$ setzt; in log. benneru leperi liegt wie in seneghe Assimilation vor, dagegen bewahrt Cagl. -uri, Sass. hat ari; ebenso hat Cagl. marmuri gegenüber marmaru der andern Dialekte.

D' Ovidios Aufsatz über den Dialekt von Campobasso (Arch. IV I 45 ff.) und Finamores Voc. dell' Uso Abbr. geben zu wenig Material, als dals ich es wagen möchte, daraus etwas zu schliefsen. Da ohnehin von D'Ovidio ein gröfseres Werk über die Dialekte der Abruzzen in Aussicht steht, glaube ich um so eher darüber weggehen zu dürfen. Ich wende mich, da die römisch-umbrische Gruppe für die Synkope vom Toskanischen kaum abweicht, sogleich zu diesem, dem italienischen $x \alpha \tau^{\prime} \xi^{\prime} \xi o x y^{\prime} \nu$.

${ }^{1} \mathrm{Zu}$ senex senecis vgl. Corssen II 207. 
6. Italienisch. Ich . weiche hier, aus Gründen der Klarheit, etwas von dem bisherigen Schema ab. Ist der Schlufskonsonant ein Verschlufslaut, so tritt bei anlautendem alten einfachen $l$ stets Synkope ein: salce selce felce elce tralce pulce ${ }^{1}$ falta sciolto volto scianto colpo polpo (caldo) soldo saldo, bei ll unterbleibt sie pollice; cavalco geht nicht auf cavallico zurück, sondern ist von den endungsbetonten Formen übertragen, wo vor dem Accent entwender schon im Lat. (vgl. mamma mamilla, búcca búccina bucína, farris farìna) oder erst im Ital. (vgl. Arch. II 343 und caratello vanello strapazzare puledro u. a.) die Doppelkonsonanz vereinfacht wurde; ebenso bei $l=r$ : alido valico; bei $r$ schwankt die Darstellung, sehen wir von den alten lardo verde $\mathrm{ab}$, so stehen sich gegenüber: varco ergo porgo scorgo chierca pirchio (merto selten und poetisch) sorco sorcio und merito larice murice sorice sen. orice, mit andern Worten $r c^{\prime} r g^{\prime}$ wird vermieden, sorce ist eine Kontaminationsbildung aus sorco und sorice; ergere ist erst von ergo aus nach dem Muster von leggo leggere gebildet, ursprünglich flektierte man ergo ereggere; die Ausgleichung ist sehr alt. - Über pancia vgl. unten. - Bei anlautend $s$ wird synkopiert: vasca brasca rasco tosco tasto sesto incastro innesto desto pesto; rs: rovisto pesca; sp: oste cesto; aber bei sc': crescito mesciko. 2 In bussola (pyxida Caix, Voc. Io Anm. 2, Studi 17) ist die Suffixabbiegung älter als die Synkope, vgl. aber busta. Nebeneinander stehen risico risco rischio; alle drei gehen von den endungsbetonten Formen des Verbums aus, da sich nur dort $i$ aus $\check{e}$ erklärt, fallen also streng genommen aufserhalb des Bereichs dieser Arbeit. Eine zu erwartende Form * resco wurde unter Einflufs von riscare zu risco umgestaltet; risico lälst sich etwa so erklären, dafs man zur Zeit, da die Synkope eintrat, zwischen résico und riséco schwankte, daher dann als letzteres riséco blieb, wăhrend erstéres zu ressco wurde; dazu lautete der Inf. risicare. Endlich rischio könnte sich zu risico verhalten, wie chian. ordio zu orridus, es könnte aber rischiare auch auf * reseculare zurückgehen. Anlautend $n$ : vanto sgomento comincio. 늘ico wird $n^{\prime}$, dic Beispiele sind magnare (nicht toskanisch, wo manico manicare oder das frz. mangiare herrscht), was auf altes * mándico zurückgeht, fogna Ascoli, Arch. III 89 Anm. 2, gragnolare scheint auf * grandiculare zurückzugehen, was aus *graniculare unter lïinfufs von grando gebildete sein kann; darf man aber ein Thoma grand- statt grandin- annehmen (vgl. galigare zu caligo) so genügt graniolare ${ }^{3}$, grugnare grundicare; endlich rogna.

- Calice ist ein Wort der Kirche, kommt also nicht in Betracht.

2 Was Storm Rom. V 174 Anm. über die Behandlung von zat im Ital. sagt, ist unrichtig.

- Mirisch Suffix -ŏlus S. 18 geht vom Subst. gragnuola aus, das ihm Ableitung von * granea ist; dieses aber (frz. grange) hat durchaus andere Bedeutung; wenn, was ich bezweifle, *granium oder * grania Kollektivum zu granum sein' könnte (man erwartet eher *granamen oder granalia), so hat doch rlus, soweit ich es übersehe, nie solche Bedeutung; gragnuola ist postverbal. 
Diez erklärt letzteres durch rubiginem, was lautlich unmöglich ist; aus rodicare entstand ${ }^{*}$ rondicare (frz. ronger G. Paris, Rom. X 59), daraus rognare und das Subst. rogna. Gegen diese Etymologie, die von blofs italienischem Standpunkte aus lautlich und begrifflich kaum angefochten werden kann, spricht rum. $r \hat{j} j e$, dessen Identität mit rogna von Miklosich, Rum. Lautl. IV 6I mit Entschiedenheit gegen Cihac, Litbl. 1882 Sp. 113 festgehalten wird, rîje beruht auf rinje, das aber nicht auf rondica zurückgehen kann (die abstufende Flexion von mandicare, wie sie noch heute in der Moldau vorkommt, lautet mänînc mancá nicht măjä). Miklosich setzt ronea an, allein was ist das? Trennt man das Wort vom Italienischen, so kann man zwar nicht mit Cihac magy. rüh zu Grunde legen, wogegen mrum.

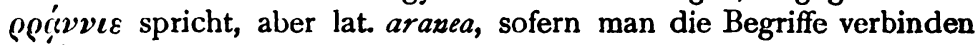
darf. - Sprach man nun aber mándica magnare oder mágno mandicare oder von jeher magnare? Unmittelbar vor dem Accente werden im Ital. Doppelkonsonanten vereinfacht, werden einfache tonlose tönend, fallen tönende aus; es konnte zu einer Zeit, da sonst $\underline{v} c_{-} \underline{v}$ noch bestand, im Nexus -dic- durch Assimilation -digentstehen, und dieses $-g \underline{v}$ dann zugleich mit dem alten $g$ ausfallen. Dies scheint mir die einzige Möglichkeit. Man beachte noch, dafs -aticum nur als Suffix -aggio giebt, -atico im Stamme bleibt. Caix, Orig. S. 249 und 252 hält das Suffix wie auch eggiare für französisch, verweist aber in den Nachträgen S. 477 auf Ascoli, Arch. 177 Anm., wo jeder Gedanke an Entlehnung fern liegt. Die Differenz zwischen natica und viaggio ist auffallig, kehrt aber im frz. nache voyage wieder, so dafs mit Caixs Annahme die Schwierigkeit nur verschoben nicht gelöst ist. Bekanntlich werden Laute in Suffixen nicht selten anders behandelt als im Wortinnern, die Gründe sind verschiedener Art. In unserm Fall hat der Umstand, dafs bei Suffix -alicum der Accent des Wortes auf mehrere Silben verteilt ist, zu folge, dals $t$ zu $d$ und das noch weiter abliegende $c$ zu $\gamma$ geschwächt, d. h., da Konsonant den Vokalen möglichst angeglichen wurde. In médico natica pedica, wo dem Nexus dic tic nur eine tontragende Silbe vorausgeht, ist dies nicht der Fall. Da hier die Synkope nach dem Accente stattfand (Verbalformen wie viuggiare sind zu selten als dals sie hätten Einflurs üben können), so wird man nicht für magno u. s. w. dieselbe Erklärung annehmen. Anders liegen die Dinge bei eggiare: aus blánkico konnte bianchio (3 silbig) aus blankéco kaum bianchéo entstehen (vgl. das Sicil. und das Span.), woher aber $g^{\prime} g^{\prime}$ ? Von den Fällen für hiatustilgendes $j$, die Diez I $178 \mathrm{f}$. annimmt, ist keiner beweisend: veggia ist *vehia, struggere tragge stehen unter dem Einflufs anderer Verba, scarafaggio lautete allerdings einst scarafajo, das $j$ ist aber berechtigt; wir dürfen nicht die "gelehrte" lateinische Form scarabaeus zu Grunde legen, sondern eine volkstümliche Gestaltung des griech. ${ }^{*} \sigma x \alpha \rho \alpha \beta \alpha t o s$, worin $\alpha \iota=a i$, $a j$, vgl. Aï $\alpha c$ Ajax. Die richtige Erklärung hat jetzt Schuchardt, Litbl. I 884 Februar gegeben; es sind Verba auf idjare, die die alten auf -iare (die doch wohl z. B. 
auf dem angedeutelten Wege aus icare entstanden sind) beeinflussen; dileggiare, von Tobler, Zeitschr. III $476 \mathrm{mit}$ den von Flechia, Arch. II 319 besprochenen Verben verglichen könnte erst aus*dilicare gebildet sein nach dem Muster von albicare - albeggiare u. s. w. Endlich erwähne ich laveggio; libeticum wurde durch Volksetymologie an lavare angelehnt, in *lavelicum aber -eticum als Suffix wie -aticum gefühlt.

Anlautend $m$ : limile fomite gomito; $b$ : sabato debito dubito subito neben malatto (vgl. Caix, Giorn. d. f. r. II 7 I, heute malato mit Suffixvertauschung) dottare detta; die drei letztern sprechen dafür, dafs die Synkope das regelmälsige ist, dafs also sabato entweder als Wort der Kirche unangetastet blieb, oder $v b a t \_$anders behandelt wird als $b u t$, und dafs die übrigen gelehrt sind. Im Nexus $b-c^{\prime}$ aber bleibt der Vokal: sovice subex. ${ }^{1}$ Endlich $v \underline{v}$ t: lievito und gavata ital. gola und gavelto = gavita mit Suffixvertauschung. Iine alte Form gaveta wurde entweder zu gavita gauta (vgl. avica ausa) oder zu gavala, welch letzteres in späterer Zeit zum teil wieder zu gavila. abgeschwächt wurde. - Synkope erscheint bei $m p$ in conto, bei $g n$ in contare; conte mag durch Proklise zu erklären sein. In prete * praebyter liegt Ausfall des $b$ resp. $\beta$ vor. lindo wird mit limpidus zusammengestellt, allein die Länge des $i$ ist im lateinischen durch nichts erwiesen, die grölste Wahrscheinlichkeit spricht für Kürze, ebenso die berg. und rum. Form; die Assimilation des $n d$ zu $n n$ im Sicil. (S. 2 I I) weist auf altes $n d$ : ich sehe darin ahd. lindi. Besondere Beachtung verdient der Nexus cit und die Behandlung von auslautendem $d$. Was letzteres betrifft, so unterbleibt die Synkope bei ripido ruvido (rugzidus Förster, Zeitschr. III 259) muffido und bei mehrfacher Konsonanz im Anlaut: torbido (mit der Rückbildung torbo, ähnlich cando, schon Vulgärlateinisch Schuchardt, Voc. II 434) morvido, sie tritt ein bei anlautender Labialis in ratto culretta, Dentalis: netto putto; die Annahme t-d könne zu zz werden ist falsch, puzzo ist *pritidium, puzzare putidiare; mozzo stammt nicht von mutidus, wie Baist, Rom. Forsch. 1108 meint; mit besserm Rechte hatte Canello 4 Jahre früher auf mutius hingewiesen, das wir, wie bassus, nur als Eigenname kennen Arch. III 328. -gzd- wird, wie gd, zu $d d$ reddo; madia aus maida weist auf griechische Betonung $\mu \alpha \gamma i \delta \alpha$ sicil. maidda, dazu stimmt der Ausfall des g. cid zeigen fracidus sucidus acidus mucidus = ital. sozzo lazzo muzzo mit tönendem $z$, somit gegenseitiger Assimilation. Daneben stehen sudicio heute auf der ersten Silbe betont, Wörterbücher aus dem Anfange unsers Jahrhunderts geben súdicio und sudicio an; fradicio und frazio. Aus sucidus entstand sudicus (frz. sourge), mit $-i$ sudicius, woraus sp. sohez (C. Michaelis Studien S. 226; G. Paris, Rom. VII ro4) ital. sudicio; Zurückziehung des Accents bei den Ableitungen auf -ius findet

1 Lat. subiex subicis, eine ganz regelmälsige, übrigens aus Ennius überlieferte Bildung, wie obiex obicis; Caix, Studi 582 sublicium verstölst gegen alle Lautgesetze. 
auch sonst statt; frazio ist nach bekanntem Gesetze aus fraizo entstanden, dieses weist auf frakitius, die Verhärtung des $t$ beruht auf Suffixverwechslung oder auf Assimilation. In sozzo, um nochmals darauf zurückzukommen, trat Synkope ein, als $k$ schon zu $k y$ geworden war, in folge der Assimilation an den Dentalen ging dieses $k y$ nicht wie sonst im Italienischen in $\check{c}$ sondern in $t y$ über, $t y+d$ wurden zu gedehntem $d y$. Ist $c$ gedeckt, so unterbleibt die Synkope und $d$ fällt: marcio moscio rancio foscio; in Neubildungen bleibt es spurcido sbiancido vincido, bei einfachem $c$ : mucido.

Endlich $c+t, c+r$. Es sind folgende Fälle: piato vuoto fate dite coto colare arruoto fare dire durre (vgl. Flechia, Arch. IV 37 I); älter sind Formen wie piaito. Flechia a. a. O. nimmt ohne weiteres * plagito plajito an, auch Ascoli, Arch. I 30 scheint sich die Sache ähnlich zu denken. Allein Ausfall von ${ }^{\circ} . c^{v}$ ist im Ital. unerhört, wenn Horning, Zur Geschichte des lat. C S. 123 faemo dafür anführt, so verkennt er, wie an mehreren andern Stellen seines sonst guten Buches die Entstehung der verschiedenen rom. Formen des lat. facere; Saraini, in den altital. Prosaromanen die gewöhnliche Form statt Saracini, ist ein Exemplum sui generis. Dafs aber piato nicht aus altem plactum entstehen kann, versteht sich von selbst, auch wenn plaito nicht vielfach bezeugt wäre. piaito (dessen zweites $i$ nicht durch Dissimilation gefallen ist, wie auffäligerweise Ascoli, Arch. I 8 $\mathbf{r}$ Anm. I lehrt) steht auf gleicher Stufe mit frz. fait: wie nun dieses durch die Mittelstufen faxto fajto entstanden ist, so können wir piaito zunächst auf piajto pia to zurückführen. Sobald wir nun annehmen, die Synkope des lateinischen $i$ sei erst eingetreten, als factum sehon zu fatto geworden war, so hindert nichts, zu sagen, ein erst in späterer Zeit mit $t$ zusammenstofsender gutturaler Explosivlaut (die Artikulationsstelle ist nicht mehr auszumachen, sie mufs naturgemäls viel weiter vorn gewesen sein als bei $k a$ ) wurde zu gutturalem Reibelaut.' Ob coto cogito arruoto adrogito braitare dito digitum ebenso $\mathrm{zu}$ erklären sind, ist zweifelhaft, da Ausfall von ${ }^{-} g^{v}$ zwar meist vor dem Accent (strega-striazzo, doch auch stria legge-leale guaina paese sactta niello reina neben regina ${ }^{2}$ ) zuweilen aber auch nach demselben vorkommt. Ausnahmen sind gracido recilo letzteres wohl gelehrt, ersteres vielleicht unter Einflufs der endungsbetonten Formen: in $\_\sim$ konnte Synkope eher eintreten als in $\llcorner\cup \perp$. - Auslautend $g$ erscheint in spargi, und im kelt. vcrtragus veltro: entw'eder wurde dem fremden Worte einfach die Endung abgeworfen (*vertrus statt vertragus) oder $\because g$ * fiel aus und ao wurde $o ; b$ : Giacomo (daneben mit Assimilation und Metathesis: Japeco, Cron. Per. I 163, 194, 235 u. s. w. Cron. Sic. 131, 165) canova; p: vescovo principe (prince ist heteroklitische Bildung). -

1 Im Span. werden wir demnächst etwas ähnliches treffen; sonst denke man an die Behandlung alter ct im Iran. Germ. Kelt. (skr. juktam iran. juxta lat. noct- got. nahts ir. in-nocht.

${ }^{2} r e$ ist wohl eine nom.-vok. Form $=$ rex; dals es nicht * rei lautet, besagt nichts dagegen (so schon Canello). 
Schliefsende liquida $(n, m, r)$ hindern die Synkope: -aggine -uggine -iggine elemosina femina frassino rondine lesina resina redina macina fiocina amoscino cecino giovane lendine cofano abrotano cotano Modana sedano telefano $u$. a. Weit verbreitet ist ferrana, das in seiner Bedeutung mit farrago übereinstimmt; es lälst sich aber weder im Ital. noch in den andern Sprachen darauf zurückführen, sondern ist eine Neubildung von far mittelst Suffix -ana. giallo ist eine Kontamination aus galbinus und gelo. Nicht klar ist tarma tarmola, letzteres könnte sich zu tarmina $(\mathbf{N}$. pl.) verhalten wie gramola $\mathrm{zu}$ * carmina (sofern die Etymologie richtig ist) wie calabr. rannula zu grandine, $\mathrm{d}$. h. es wäre das Gegenstück $\mathrm{zu}$ modano aus modulus; tarma kann eine Rückbildung sein, oder das Fem. zu tarmus (Isidor). Anders Ascoli, Arch. IV 400. - Unrichtig ist die Herleitung von frana aus fragmina, doch weifs ich keine andere; über rogna vgl. S. 215. Schon lat. ist die Synkope in donna. In furno -arno -irno u. s. w. haben sich die beiden Liquiden angezogen.

Bei $-m$ unterbleibt die Synkope ebenfalls: animo (almo poetisch aus dem Prov.) decimo attimo spitama pittima lagrima edima, nur bei anlautend $l r$ tritt sie ein: calmo ermo, bei $s$ ist sie fakultativ: bias(i)mo (der Mangel des $\varphi$ weist auf eine einst synkopierte Form) medes(i)mo, vgl. Cosimo. Jung ist maremma, da altes $t m$ zu $s m$ wird (arismetica).

Auch bei $r$ verhält es sich ähnlich, ovra ist poetisch und stammt aus Frankreich; sonst sind nur lepra und burro zu nennen; ferner die Infinitive von Verben auf kere, vgl. S. 217; man pflegt fare und dire durch Anlehnung an die übrigen Verba zu erklären, allein dann mülste auch durre so gefalst werden. Überdies giebt es im Praesens von facio und dico keine einzige Form (abgesehen von 2. Sing. impt.), die lautgesetzlich mit Formen von amo oder parto zusammenfiele. Entstand aber aus fakere faxre farre $(\alpha$ assimiliert sich folgendem $r$, wie $l$ in sciorre, daher die Differenz zwischen *farre-piato) so lag es bei dem vielgebrauchten Verbum nahe den Inf., sodann das Präsens nach amare umzuformen; ebenso ging es bei dire, während bei dem älteren trarre und vollends bei sciorre durre u. s. w. eine solche Attraktion unmöglich ist.

Es bleiben noch einige zweifelhafte oder falsche Etymologicen. It. gonzo sic. gunzu stellt Baist, Ztschr. VI I 35 Anm. 2, vgl. Rom. Forsch. 1 I 7 mit caudex zusammen; jedenfalls ist von codex auszugehen, ital. $o$ ( $g$ und $g$ sind bei etymologischen Untersuchung zu scheiden; Baist pflegt freilich auf solche Sachen nicht zu achten) sic. $u$ verlangen $o$, laut. au giebt $g$; aber auch $g=c$, der durch nichts motivierte Einschub des $n$ sind auffälig. Ist die Etymologie doch richtig, so ist entweder das $n$ jünger als die Synkope oder $n d^{\prime \prime} c^{\prime}$ wurde zu $n z$, welch letzteres wenig wahrscheinlich ist, da bei $c^{\prime}$ Synkope so selten ist. - Auch mit monco muticus Baist, Ztschr. a. a. O. kann ich mich nicht befreunden: $g$ widerspricht, ich möchte vorziehen was Diez, Wb. I darüber sagt. - bravo = rabidus bespricht Storm, Rom. V 170 (von Scheler bei Diez Wb. ungenau 
wiedergegeben): mit Vorschlag eines $b$ entstand brabido braido 1 oder bravio bravo; dabei ist nur das hypotetische bravio wegzulassen, bravo entstand durch Abwerfung der ganzen Endung. broncio $=$ raucidus Baist, Zeitschr. V 558; Caix, Studi 12 hatte schon 1878 das Wort in anderer Weise besprochen, noch mehr hat die von G. Paris, Rom. VIII 618 angedeutete Erklärung für sich.

Der tonlose Vokal ist gewöhnlich $i$; daneben $a$ vor $n$ : cofano abrotano cotano modano Modana (toskanisch) gicvane sedano pampana garofano ebano u. s. w. Mussafia, B. 12 Anm. 4; Caix, Vocal. 26, vor m: Girolamo Bergamo attamo (heute attimo vgl. Canello, Arch. III 335), vor $c$ : monaco cronaca Monaco indaco sindaco mantaco; $g$ : folaga prolago astrolago; $f$ : orafo; meist, nicht immer, ist der betonte Vokal $o$; abgesehen von giovane, welches vielleicht durch ein altes giovano beeinflufst wurde, ist der Aulaut $o$, bei $e i$ steht $i$ vgl. modine-modano; abrotine-abrotano; folice-folaga, ebenso bei anlautender Palatalis: fiocina amoscino cecino. - o: menomo.

Schwieriger liegt die Sache bei $r: a$ wird, wie vor dem Tone, zu e: gambero zucchero cetera bacchera nattera Gaspero Lazzero; ebenso $i$, vgl. Caix, Voc. 9. Statt $o$ aber erscheint teils $e$, teils $o$, vgl. Caix a. a. O., die Pl. auf -ora, die meisten $r$-Stämme (lat. $o$ und $u$ ) behalten es; aber rovere albero cantera (nach Caix ein singularisierter Plural) zeigen $e$. Die Erklärung, die Caix giebt, beruht auf einer durch Corssen verschuldeten ganz falschen Auffassung der lat. Verhältnisse. In den zwei ersten Beispielen kann man sehr alte Anlehnung an die Flexion auf $u(s)$ eris (aus robur war in Italien frühe robu wie aus genus genu entstanden) oder an die ital. Bildungen auf ero -ere sehen. Die vielen Fälle von ero bedürfen einer eingehenden Untersuchung; merkwürdig ist, dafs ero nicht selten an die Stelle von olo tritt, doch bleibt auch hier zuweilen o: spillonzora lopporo lontoro (man beachte das $o$ im Stamme); dürfte man annehmen, die betreffenden Wortc seien aus den keltotoskanischen (man gestatte mir diese administrative Verhältnisse mit ethnologischen verbindende Bezeichnung) Dialekten nach Florenz gekommen, so wäre der Sache geholfen: dort wird olo zu elo, im rein Toskanischen entstand daraus entweder olo oder cro. Allein eine solche Hypothese läfst sich kaum irgendwie wahrscheinlich machen; eher lälst sich sagen, dafs alte Bildungen auf -ero (deren Ursprung sehr verschiedener Art ist) eine Anzahl derjenigen auf -olo, in welchen der Begriff des verächtlichen lag oder gelegt werden sollte, anzogen. Dafs $l$ in $r$ überging und dann unter dem Einflufs des $r$ das tonlose $.0 \mathrm{zu} e$ wurde, glaube ich nicht, weil der Fälle von erhaltenem $o$ vor $r$ genug sind; sehen wir von folgore tortora, bei denen die erste Silbe Einflufs übte, ab, so bleiben namentlich die Pl. auf -ora, die z. B. im Montal. noch heute gebrüuchlich sind (prat. ram. pugn. arc. Nerucci) ${ }^{2}$, ferner ancora. - Dafs die Be-

${ }^{1}$ Caix, Studi $218=$ bereit, wenig wahrscheinlich.

2 Hier eine Anzahl solcher Formen aus altital. Texten; nicht aus Tosk., 
handlung des tonlosen Vokals vor $r(e$ oder $a$ ) ein wichtiges Unterscheidungszeichen der verschiedenen toskanischen Dialekte ist, ist bekannt, und braucht hier nicht weiter ausgeführt $\mathrm{zu}$ werden.

7. Emilianisch. Ich lege das Romagnolische (nach Morri und Mussafia, Wiener Sitzber. B. 67 S. 653 ff.) zu Grunde. Hier ist das Grundgesetz: Synkope tritt stets ein, wenn es die umgebenden Konsonanten gestatten, d. h. immer bei den Fem. auf $a$, bei den Masc. nur, wenn der zweite Kons. ein Verschlufslaut oder ein Zischlaut ist; ist er sonant, so entwickelt sich der Stimmton und zwar als $u$ vor $m$, als $a$ vor $n r l$. Die Synkope ist jünger 1 . als die Erweichung intervokalischer Tenues, daher edgh (aticus) edgha cergh tosg tisg dmeng mangh manga perdga tonga cargh cargha bol. pondgh mod. pondeg, wo $e$ erst durch Svarabhakti entstanden sein mufs, scorga sorgh tsevd tevd ruvd trovd grevd omd lend, vielleicht auch cand ranz mez levd, nur im Nexus lzp ist der Ausfall des $\check{z}$ älter fulp; 2. als die Assibilation: mesna piantasna tussilazna calezna fulazna sflezna rezna (woraus ein Adj. rezan; über die Formen auf -agen: balurdagen daben buls u. a. vgl. S. 2 I I). Da neben einander stehen êsan êsna biêsum (aus *biêsm) chesca mesna cataplesma einerseits, azna andererseits, so folgt daraus, dals aǵin vor, asin aćin nach Übergang des $a$ in $\hat{e}$ synkopiert wurden. Sonst haben wir noch ponsa polsa pänza urebs romsa zemsa; aus der Schriftsprache sals. In gomt (cubitus) hat die Synkope früher, in gomit (vomitus) lemit gar nicht stattgefunden; ospilem giebt ost, digitus di. Adj. auf -zdus: bei anlautender Doppelkonsonanz bleibt der Vokal und fällt $d$ : morbi; die ganze Silbe bleibt im jungen repid und in lozzid temid lepid lequid azzid. Sollen die letztern alle Lehnworte sein? Beim dritten spricht $p$ beim letzten $a$ für, bei den übrigen $o$ und $e$ nicht gegen diese Annahme. Auslautend $n$ : abrotan cherpan ergan sflosna frassin petan merzan vartezin lesna, $m$ : anma endma aber legrima. Das letztere zeigt, dafs merzan nicht aus * merz' $n$ entstanden sein kann, oder, falls heute bei vokalischem Anlaut des folgenden Wortes merźn gesagt wird, dals die Synkope im Nexus $r g^{\prime}$ in später eingetreten ist, als im Nexus gin, d.h. nach dem Übergange von $a$ in $e$.

Mussafia glaubt ( $\S$ III) a bleibe erhalten und führt dafür stomach monach an; in pêlgh sparz canev sei $a$ zunächt zu $e$ geschwächt worden, wie im venez. sparesi canevo, dasselbe oder Verdunkelung des $a \mathrm{zu} u$ habe stattgefunden in belsum dettum, auch in landa sei $a$ zunächst geschwächt worden. Allein das Venez.

aber doch aus Gebieten, wo $r$ nicht $o$ vor sich bedingt: Hist. Rom. frg.: loc. 1,$1 ; \operatorname{arc} 1,1 ;$ pec. 1,$3 ; 1,9 ; \operatorname{corp} .1,3 ; 1,9 ;$ fic. 1,$5 ; 1,13 ; \operatorname{cap} .1,9$; corai. 1, 12; Cola d. Rienzi cap. 419, sonn. 431 (neben gewöhnlicherem suonni) foc. 455, vent. 437, corp. 437, fic. 455, clin 483. Chron. Aqu. foc. I 87, colp. I I I, quart 309, II 733, loc. II 407, nom. I 683. Doc. Per. nom. Arch. Stor. XVI 2, das Trat. d. Masc. schwankt zwischen $u$ und $o$, dem Charakter der Mundart entsprechend: corai. 3, adiut. 3, corp. 5, 73, temp. 29, lat. 59, flanc. 9, 129, coi. 45, loc. 17, 29 (-i 71) cib. 49, fic. 53, cap. 89, viai. 101, amnura le pulaura 181. nomera 211. - Pisanisch: lat. (Arch. Stor. VI 2) 97, grad. 122. 
bcweist fürs Romagnolische nichts, da dort alle nachtonigen Vokale zu $e$ geschwächt werden; monach und stomach können viel eher Worte der Schriftsprache sein, namentlich ersteres; in den übrigen ist allerdings Schwächung des $a$ zu $e$ eingetreten, aber schon zu einer Zeit, da die alten Nachtonvokale noch bestanden; dieses neue $e$ fiel gleichzeitig mit dem aus $i$ entstandenen aus.

8. Lombardisch. Ich beschränke mich aufs Mailändische (vgl. Salvioni, Fonetica del dialetto moderno della città di Milano S. yo ff.), Mantes endes erpes fires forbes rümes sales (und mit Assimilation salas) guides serpes polles pures pomes scimes tripes (atriplex) tros aus $\operatorname{tra}(d) u c e(m)$ (vgl. Salvioni $\S 0_{4}$, dessen Ausführungen ich durchaus beistimme) - panscia; manega panzanega voladega domenega monega endegh medeg perlega $\mathrm{u}$. s. w. Synkope tritt, vor der Lautabstufung, bei anlautendem $s$ ein: brasca rasca vgl. ascass, dagegen ist trienza nicht, wie Salvioni $\S 176^{\mathbf{b}}$ meint, * tridentica sondern trident-ia; ebenso remonzd * remundiare (Flechia, Arch. IV 375) nicht * remundicare (Salvioni $\S 319^{c}$ ) aticus *adegh, daneben entlehntes $a g^{\prime} g^{\prime}$. formaj möchte ich für ein dem rätischen stratum angehörendes Wort betrachten. Denn auch in Mailand lebte ähnlich wie in Venedig, nur noch mehr im Dunkel der Vorzeit verhüllt, rätisches Element neben lombardischem, und dals ein Wort, wie jormaj, das vom rätisch sprechenden Lande bezw. den Bergen in die lombardische Stadt kam, zunächst seine alte Form ( ${ }^{*}$ formadi) mit schwacher Änderung behielt, ist nicht unwahrscheinlich. Zwar sagt Gartner $\S 6$ caseolus sei die echt rätische Form, formaticum in centralen und östlichen Mundarten stamme aus venedischem Munde; allein formádi ist so durchaus rätisch (vgl. Ascoli, Arch. I 75 viadi), dafs es mir wahrscheinlicher ist, an denjenigen Orten, wo formádi und viás nebeneinanderstehen, sei viás entlehnt. Bei anlautend $l$ $r n$ schwankt die Darstellung carga erga melga gringa - čerega folega, $n d \tilde{i c}$ giebt wie im ital. $\tilde{n}$ mañd foñd remoñd. - ameda und mit Accentverschiebung meda ${ }^{1}$ aneda derpeda debet gombet limet prevet messeda caved; Synkope tritt nur in Vortonsilbe ein und ist von da zum teil in die stammbetonten Verbalformen eingedrungen; nebeneinander stehen pondd ponta, das erstere aus ${ }^{*}$ poneda (von póneda 3. Sg.) unter Einflufs des letzteren. In crella haben sich die zwei Dentalen angezogen, vgl. unten. $g$ : regöy (recolligo) sparg'. - $d$ nett (frĕ̌ cald); canded lamped ledegh (liquidus) luzed smorbed (neben morbio bei Bonvesin, etwa unter Einflufs des Abstraktums: morbiezza) tresped leved amed und die Neubildungen slegned zerbed strimed; die Fem. lauten -eda. Ausfall des $d$ zeigt ranš marš, frapp; litta $=l i-$ quida (Flechia, Arch. II 325) ist zweifelhaft, ratta. - $n$ : asna masna bresma frosna lesna limösna carisna, vgl. purisnd fasnd desma; aber incusgen calusgen u. a.; cöden ${ }^{2}$ u. a., provanna ist *propana statt pro-

1 Daraus mit bei einem Koseworte gerne eintretender Assimilation dedo im judic. und in lomb. Dialekten.

2 cōs cotis stimmt zu südit. cutula, nicht zu mail. coeden, welch letzteres auf abstufende Deklination cōs * cőtis cottinus weist. 
pago (Salvionis Erklärung löst die Schwierigkeit nicht; ebensowenig Flechias, Arch. II 373 ff.), herbeigerufen durch * farrana statt farrago. Merkwürdig ist cartelamm cartilago, gewissermafsen cartilamen. Bei $r$ ist Synkope Regel: šendra cambra tender u. s. w. Salvioni 103.

Der tonlose Vokal ist meist $e$, selten unter labialem Einflusse - vgl. die Beispiele für letzteres bei Salvioni S. 127. Dic Bemerkung, es sei dieses $e$ einer der charakteristischen Züge des Mail. ist nur richtig, wenn man die Schriftsprache, nicht wenn man z. B. das Venez. vergleicht.

Geht man von Mailand dem Fufse der Alpen zu, so mehren sich die westrätischen Elemente zusehends. $\mathrm{Da}$ das Westrät. gerade in der Behandlung der Nachtonsilbe im direkten Gegensatze zum Lomb. steht (es wirft den Vokal meist aus und nähert sich hierin, wie in andern Punkten, dem Emilianischen), so wäre eine genaue Angabe für die einzelnen Worte in den einzelnen Gegenden sehr wünschenswert. Ich verfüge leider nur über das im Arch. Gl. I gebotene Material und über die Wörterbücher von Monti und Tiraboschi; eine Aufzählung der wenigen darin enthaltenen Formen könnte, wegen ihrer Unvollständigkeit, nicht vieles lehren; für die Hauptsache mögen ein paar Beispiele genügen. Im Bergamaskischen findet sich codga domenga lüganga bosga, sonst stimmt es ganz mit dem Mail. überein; wenn graida und gravia neben einander stehen, so ist das letztere westlomb., das ersterere ostlomb., wie auch oladega, das bis nach Mailand vordringt, letzterer Gruppe angehört.1 preit nicht preb(e)ter sondern pre(b)iter vgl. prevt im Voc. lat.-berg. calösen neben cali letzteres westrät., so wohl auch canva neben canea (vgl. caenva Val. Anz., Arch. I 254). Auch zumna wird rät. sein.

9. Das Venezianische meidet die Synkope, obgleich es alle auslautenden Vokale erhält, wodurch es also noch viel mehr „daktylisch" wird, als das Toskanische.

pulese polese mantese cimese felese elese salese larese pomega pulega melega lendego fontego folega chiavega persego risego stomego, vgl. spareso (wegen des $s$ vgl. Salvioni a. a. O. $\$ 367$ ); pre vodo comio; folpo; tivio torbio morbio granzio; lampeda cospedo domada - marzo; canevo; lentizene cavezina ancuzene u. a.; gendena fossena frassenc piadena carpano; calmo. - Ferner verzeichnet Boerio aus Chioggia sperga = pertica, eine trotz des vened. s- unzweifelhaft rätische Form, auffällig ist dabei nur, dals sie westliches nicht östliches Gepräge trägt. - Vor $r$ bleibt $e$ çénere u. a.

Vokal ist stets $e$, auch wo lat. a steht; Ausnahme das alte domada und carpano mit Assimilation.

I0. Auch das Genuesische (nach Olivieri) weicht wenig von diesem Typus ab: embrexu dentexu endexu remexe - aber $l(i) c^{\prime}$

1 Abfall von $v$ - und Ausfall von $v v v$, wo es die übrigen rom. Sprachen behalten, teilt das Ostlomb. mit dem Vened. im Gegensatz zum Rät. und Westlomb. Soviel mag hier genügen. 
$r(i) c^{\prime}$ : erxu feci prüxa saxi; sinica endegu fullega teuscigu fundegu marega persegu -aegu $=a(t) i c u(m)$ naeghe vaegu $=$ var(i)go neben merga; diu (digilus) ciaetu (pla(c)ztum; purpa marsu (marcidus) ransiu rüdegu (aus *rugidus); canea; -aze, unchizze rüzze caize carpe, proanha fusana - lendena; beuzma. Intervokalisches $l r$ fällt im Genuesischen, wodurch wir einen Anhalt für Zeitbestimmung der einzelnen Fälle bekommen. Auch wo $r$ am Schlusse der Nachtonsilbe steht, geht es verloren: senee (ciner) seixau (cicer) zeneu (gener) dezeuviu (* deex-operum) peivie; auch zenevau sagau (sargo *sarago * sagaro) pagau $(\pi \dot{\alpha} \gamma \rho 0 s)^{\prime}$, aber levre, wo also die Synkope alt ist (vgl. das Ital.); auch bei aus $l$ entstandenem $r$ : dattau nespua tellua lodua. Während in diesen letztern Fällen der tonlose Vokal $u$ ist, finden wir dagegen bei altem $r$ (wozu im Grunde auch dattau gehört) teils, wie vor den übrigen Konsonanten $e$, teils $a$, ohne dafs sich eine Regel finden liefse. Man sieht nur, dafs auslautend $-u$ im allgemeinen $a,-e$ liebt.

I I. Piemontesisch (vgl. Ascoli, Arch. II II9 Anm. I; nach Sant' Albino) cimes feites pules mantes poles pomes, stomi perssi mani tossi mania melia monia pertia; ania (anitre) gomi preive voeuid-di magna (zia: aus amita entstand amia, durch Reduplikation mamia, daraus magna, vgl. friul. agne); ranssi; reidi (so Ascoli, Arch. I 654 und Sant'Albino; Ponza reid) ruid (neben ruid) gehen zunächst auf * ridigus * rudigus zurück; terbol turbidus; marss. Auslautend $n$ wird zu $u$ : ǵư $u$ ancusu u. s. w., vgl. Ascoli a. a. O.; -na synkopiert: lendna redna lesna losna lodna limosna frosna fomna-beusma. Aber bei $-r$ unterbleibt Synkope stets, der Vokal ist e, vgl. Ascoli. Auch hier finden wir pansa und provana wieder.

Im-Rätischen ist ein tiefgehender Unterschied zwischen westlichen und östlichen Idiomen zu konstatieren: jene folgen dem span.-prov., diese dem oberital. Princip. Leider ist in Gartners sonst vortrefflicher Grammatik dies nicht genügend hervorgehoben, vgl. $\$ 6 \mathbf{r}$.

I 2. Friul. (nach Pirona, G. = Gartner) pomighe crodie domenie mani manie piertie nadie, doch bei $\dot{c}$ : pulš (pulex G.), die Zehnerzahlen: uindis dódiš u. s. w. stehen unter dem Einflusse von diš. semide sabide préli agne (amita) - plaid vuaid; moscid tivid ransid spavid rampid limpid - rctt - ruspi, aber marz; fraid wie plaid; rusin zovin fossine argane pielin uarfin u. a.; blosime. Besondere Aufmerksamkeit verdient -er-; die Infin. gehen auf $-i$ aus: vendi (G. § 167), aus vende(re); ebenso -erum: numar u.s.w. mit -er zu ar; -erem polvar, u. a. Arch. I 503; Synkope tritt nur in wenigen Fällen ein rori = robrem. etwa nach uber ubris flektiert, ob ajar pevar u. s. w. masc. oder neutr. Formen sind, läfst sich nicht sicher entscheiden.

1 Der Fisch heifst ital. parago; es wäre möglich, dals er von Genua in die Toskana gekommen wäre, wo man das fremde Wort nach dem Muster von sagau sarago umformte. 
13. Surselvisch: -adi tissi muni, aber dumendya (G.) u. a.; cudasch pules dudesch; spirt de semda onda sonda kumbel (cubitus $G$. $\$ 92 \xi)$; mezeamda; avdan; cret cretta neidi tievi - asch, ronsch aber mantsch, frigdus sollte freğ geben, das heutige frcid scheint aus dem Engadin zu kommen. söli (eng.). - semna asıa (m. asen) olma ruinna, vgl. noch Gartner $\$ 78$-tudinem und incudinem, olma dieschma.

Die tirolischen und tessinischen Dialekte zeigen oder vermeiden die Synkope, je nachdem sie mehr oder weniger das rätische Substrat mit lombardischem und venedischem versetzt haben. So ist nach P. Monti dmada comask., aber im Val. Verz. sagt man anda, caneva comask., cheuna lev. cienva Val. Maggia; giovana neben jona Val. Verz. u. s. w. - Das Judicarische, das sonst durchaus vened. Formen zeigt, hat $p v \check{s}$ sor $\breve{s}$ und furmai. In Sulzberg treffen wir wie natürlich ncben rät. amdo femma ommi ein vened. domenego poleci; ganz rät. ist das Bergell: mešna äsna manga luganga rasgär. - Eine genaue Untersuchung der „Amphizone“ in diesem Punkte wäre erwünscht.

14. Spanisch. Im allgemeinen gehen Span. und Portug. dieselben Wege, für beide Sprachen gilt als Hauptgesetz: Ist der Schlufskonsonant ein Explosivlaut, so tritt wo möglich Synkope ein nach Erweichung der intervokalischen Tenues; die Fälle, wo tonloses $\boldsymbol{\beta}$ (geschrieben $c$ ) erscheint, brauchen nicht unbedingt eine Ausnahme zu bilden, da das heutige Span. kein $\partial$ besitzt.

sauce sorce urce (ulex wie Baist Zeitschr. V 556 erkannt hat) auce (apex) I vgl. encina pulga polgar; nebeneinander stehen ponce und ein adjektiviertes (piedra) pomez, letzteres wohl halb gelehrt (oder soll man an pùmicius denken? Dann würde man das Fem. erwarten). Ist der anlautende Konsonant /, so verbindet er sich mit $i$ zu ch corche und corcho, pancho neben panza; dasselbe findet aus mir unbekanntem Grunde in chinche (dessen erstes ch an das zweite assimiliert ist) statt. codigo ist ein Wort der Gerichtssprache, añagaza = ilex Wb. 493 ist sehr unwahrscheinlich, ebenso alerce larix Wb. 419; juez (neben dem aus dem Katal. entlehnten juge) lehrt, dafs der Ausfall von $\_d$ - älter ist, als die Synkope, also wohl gleichzeitig mit dem Übergang von $v t^{v}$ in $v d v$. Aus dein Portug. erwähne ich noch lousa efa. - Tcus, a: mielga mango longa pentasirgo (-syricum Baist, Zeitschr. VII I 20) domingo galgo jarga Astorga algo crego (*crericus aus cl.) mielgo (gemellicus oder eher gemellus meint Baist, Zeitschr. V 562, das letztere ganz unzulässig, salgo was B. vergleicht, ist durchaus verschieden) fragua (fabrica * frauga) riesgo (aber risco arab. risq nach Devic, Scheler bei Diez S. 729 zweifelt ohne Grund) estantigua (vgl. Baist, Zeitschr. V 223) nalga (aus nadga), -azgo = aticum aus adgo adgo, juzgo (judicum Gen. pl.) piezgo; viele Beispiele giebt die Konjugation masgo jusgo rasgo cargo amargo otorgo cabalgo huelgo u. s. w., -guar = ficar, worüber

1 So auch Baist, Zeitschr. VI 161 . 
Diez II s. v. santiguar nachzusehen ist. - portug. merke sengo Seneca (Michaelis, Zeitschr. VII 102, besser senic-um G. Paris, Rom. XII 4I 2. - Ausnahmen: aje = aticum, meje = medicum erege hereticum, manjar, jorgina canonge forja monje, die vier letztern nach Baist, Zeitschr. VI 427 echt spanisch. Dals aje und manjar aus Frankreich stammen, ist wohl allgemein anerkannt. Entlehnung nehme ich auch für forje an, obschon Baist a.a. O. das Gegenteil behauptet: wie soll es auf demselben Boden entstanden sein, aus welchem schon fragua entsprols? auch meje und ereje tragen durchaus frz., besser prov. catal. Gepräge. Dagegen liegt in monje und dem danach geformten canonje eine andere Behandlung des griechischen $\chi$ und $x$ als des lateinischen $c$ vor, vgl. S. 213 . Was jorgina ist, weifs ich nicht; Baist hat bis jetzt zwei Ansichten über das Wort geäufsert, deren zweite wenigstens nicht ein so regelloses Phantasiespiel ist, wie die erste Zeitschr. V 244 und VII 1 22; aber auch diese (sorecina) ist, wenn man encina vergleicht, lautlich sehr zweifelhaft, und begrifflich nicht sehr überzeugend. Könnte nicht eine Ableitung vom Eigennamen Jorge zu Grunde liegen? „Meister Hämmerlî"“ (Gelehrter des XV. Jahrh.) ist bei uns synonym mit "Hexenmeister". Wer diese "Georgina“ war, dürfte freilich schwer zu ermitteln sein. - percha porche erweisen sich durch den Mangel des Diphthongs als Fremdwörter. - trigo = triticum wirft die ganze Mittelsilbe aus. -atico wird durch -adego zu -aðgo, woraus -azgo, dial. -algo. - Lautet die Silbe mit Doppelkonsonanz an, so unterbleibt die Synkope: lobrego (lubricus nach Förster, Zeitschr. III 562; Cuervo, Rom. XII 109; rubricus Baist, Zeitschr. VII 120) albegar, ferner wenn der tonlose Vokal $a$ ist pielago, portug. prego, vgl. alaga; estomago. Beide Bedingungen treffen zusammen in vástago (nach Storm, Rom. V $185=$ got. vahstus). In dem halbgelehrten lorica hat die Accentverschiebung zu einer Zeit stattgefunden, wo die Synkope längst vollzogen war; tosigo erweist sich trotz $g$ durch 0 und $i$ als Lehnwort. In mehreren Fällen scheint die Synkope älter als die Lautabstufung zu sein: focha fulica neben floja geht wohl auf ein dim. fúl(i)cula zurück, das beiden Formen gerecht wird; barca nach Diez von Bápls *barica (Wb. 42) nach Wackernagel (Haupts Zeitschr. IX 573) von altn. barkr. Das letztere wird dadurch ausgeschlossen, dars barca schon auf lateinischen Inschriften Spaniens vorkommt (Hübner, Berliner Monatsber. 1860 S. 752), von Spanien aus dringt es weiter: vielleicht ist es ein uraltes Wort; irgend eine Berechtigung zur Ansetzung eines Typus, *barica existiert somit nicht. trinca, nach Bugge, Rom. V 187 von *trinicus, Storm ib. sieht darin, und wegen des $k$ slatt $g$ mit mehr Recht, eine Rückbildung aus trinquete (trinquetrus), ganz anders Diez, $\mathrm{Wb}$. II ${ }^{\mathrm{b}}$, wo übrigens trinicus fragend angeführt wird; bei kombiniertem $s$ (das also nicht, wie einfaches, tönend wird): tosco torsicus Storm, Rom. V I 85 ; in enclenque, das von Hotmann, Zeitschr. VI 435 glücklich auf mlat. incleticus zurückgeführt ist, nur hätte das Verhältnis dieses Wortes zum gr. $c^{\prime} \gamma x \lambda \hat{\imath} \tau \iota x c_{\text {ós }}$ eine Bemerkung verdient;

Zoitsohr. f. rom. Phil. VIII. 
Baist ebenda setzt als Mittelstufen an enclelgo - enclenco, wobei der Übergang von ngo in nco unerklärt bleibt, eher wird man enclent(e)go enclenque anzunehmen haben. Aus dem assimilierenden Einflusse eines zwischen zwei Konsonanten ausgefallenen $t$ erklärt sich auch das $c$ in masco. Nebeneinander finden sich prisco und persigo, letzteres, wie $e$ und $r$ s zeigen, gelehrt, ersteres aus pirsco; endlich trocar = tropicare oder travicare, besser traucare (die Diphthongierung in den stammbetonten Formen ist ohne Belang), vgl. wegen der Erhaltung des $c$ Baist, Rom. Forsch. I 445. Aulserdem zuigen noch barcar volcar coscar esparrancar cascar $c$ statt $g$ : bei cascar sind die anlautenden Konsonanten ss, coscar $=$ coctit icare, also derselbe Grund; in barcar ist die Metathesis des $r$ älter als die Synkope, volcar aus volvcar erklärt sich wie trocar; die Etymologie von esparrancar ist nicht sicher: Diez denkt an ital. spalancare, Caix, Studi 103 expernicare; endlich span. terco tetricus *teť́rcus. ${ }^{1}$ - Ich erwähne noch span. yezgo portug. engo, die Diez zweifelnd auf ebulus zurückführt; zu Grunde liegt ęticum; man denkt unwillkürlich an deutsches "Attich“, doch ist damit nichts anzufangen, vgl. Kluge. - Aus dem Portug. nenne ich nur einige Fälle, in denen die ganze Endung abgefállen ist: toso pea. In einem Falle weicht es vom Span. ab, in der Behandlung von -dĩc-, vgl. sojo *sudicus, crujar * crodicare aus *crocidare crociture, pejar pedicare. ${ }^{2}$ - Endlich ist interessant derrengar (disrenicare) neben derrear (*disrenare), das zeigt, dals hier die Synkope älter ist, als der Ausfall von 브르. - Die Verba auf icare erscheinen im Span. in der Form ear, im Portug. ear und ejar. Häufig stehen in letzterer Sprache beide Formen nebeneinander, zuweilen entspricht auch einem portug. ejar ein span. ear, vgl.: carcarear und carcarej., gote. und gotej., serpe. und serpej., solfe. und solfej., adej. neben ale. (span. carcare. gote. serpe. ale.) amerellej. span. amarille., ebenso finden sich zu apedrej. bafej. bandej. barquej. bocej. boquej. u. a. span. Formen auf ear; einmal (boce.) hat das span. ear und ezar, mit portug. bandear. deckt sich span. bandejar in der Bedeutung. Seltener ist portug. ejar = span. ejar: azulej. cotej. bosquej. arpej. u. a. Diese letztern sind entlehnt teils aus dem Span. (-ejar = zculare) teils aus dem Ital. (-eggiare). Die echten Verba auf ejare weisen auf idiare (vgl. hoje nojo neben meio raio moio), diejenigen auf eare, wenn sie Nebenformen auf eiare haben, ebenfalls, ferner auf enare (alhe cerce pente seme): igare (fume) und zum Teil auf icare mit in den stammbetonten Formen accentuiertem $\stackrel{\downarrow}{2}$ Man ist versucht, da wo portug. ejar = span. ear ist, fürs Spán. dieselbe Erklärung anzu-

1 Baist bestreitet die Möglichkeit des Abfalls einer wiederholten Silbe Zeitschr. V 243, wie er sich das span. Wort zurechtlegt, weifs ich nicht; an cenno = cincinnus ist nicht zu rütteln, die $R$. Forsch. I 134 vorgebrachte Etymologie stöIst im Ital. auf lautliche Schwierigkeiten; vgl. sonst Caix, Studi I89f., sic. arrivisciri lat. $\bar{u} n$ ] ündeciom (von Gartner $\$ 56$ nicht verstanden) und die Reflexe von cucurbita.

2 Das Subst. pejo ist postverbal, hat also mit lat. pedica keinen direkten Zusammenhang und stimmt nur zutällig mit frz. piège im Geschlecht überein. 
nehmen: zwar wird im Span. dj sonst anders behandelt: hoi rayo bayo moyo hastio cnteyo, das $y$ mag aber, zunächst vor dem Tone, ausgefallen sein. In den Fällen nun, wo man einen Wert darauf legte, das alte Verbum auf -icare als ein abgeleitetes zu betrachten, bewirkte der Typus idịa- zunächst Accentverschiebung ว $c o$; dieses auch das umgekehrte eintritt), $-g \underline{v}$ aber fiel im Span. und Portug. aus. So ist also der Mangel der Synkope in ear = zcare aus einer alten Accentverschiebung erklärbar. Es bleiben noch die Zahlen I I ff. span. once doce portug. onze doze; auch das Span. hatte einst die zu erwartende tönende Spirans, vgl. Horning, Lat. $C$ S. ıoo, dazu José, der nur doze mit $\dot{z} e(\xi)$ kennt. $-t$ verhält sich wie $c$ : span. beodo muebdo duda codo cuende anda (amit-a, lat. ames, -itis, also Fem. nicht Masc.., wie Klotz angiebt, vgl. landa im Dialekt von Albertville) dedo cuido; pleito ist, wie pl zeigt, halb gelehrt, ebenso empleita und emplenta (zu dieser Behandlung von $c(i) t$ oder $c t$ in nicht populären Worten vgl. enteco) mpit: cuenta. Älter ist die Synkope bei anlautend $l r$ : falta vuelta suelta, bei anlautendem Dental venta (aber renta ist wegen $e$ statt ie gallicismus). Da nit nd giebt (vgl. noch das dunkle hienda und estruenda), so kann aliento nicht auf alétnitus (vgl. frz. aleine) zurückgehen, die Vertauschung der zwei Liquiden hat erst nach der Synkope stattgefunden anelto aliento, wohl unter Einflufs der übrigen Bildungen auf -iento. C. Michaelis kennt auch eneldo, wo also der Vokal richtig wiedergegeben ist. Ich denke es existierten zur Zeit, da die Synkope wirkte, nebeneinander anéltitus und alęnitus; daraus entstand anelto alendo und durch Vermischung der zwei Formen *aneldo eneldo. - Intervokalisches $p$ war beim Eintreten der Synkope noch tonlos, daher grieta retar, und natürlich contar entar. Bemerkenswert ist das Ergebnis von cit reso neben lezda licita Baist, Zeitschr. VII 120; von sit in transe; da es kein amizad sondern nur amistad giebt, so müssen im Verbum die stammbetonten Formen Sieger geworden sein; aus rezdo konnte, bei der spirantischen Aussprache des $d$ sehr leicht durch Assimilation zunächst gedehntes $z$, dann, da das Span. keine gedehnten Konsonanten (aufser $r$ ) kennt, einfaches entstehen. - Bei anlautend mehrfacher Konsonanz unterbleibt die Synkope: perdida huesped cesped; ebenso, wenn der tonlose Vokal $a$ ist: gabata sabado anade portug. adem. (In Vortonsilbe aber erscheint sie: galtera, das Wort anders zu erklären, wie Baist, Zeitschr. VI I 17 will, ist unnötig; vgl. wegen au-al calma salma cat. malalt u. a.) Das Portug. geht auch hier seine eignen Wege bei anlautend $b$ : duvida covado bevodo, sonst stimmt es mit dem Spanischen überein: quejando (Diez, Wb. 749) dondo andas u. s. w. - $p$ : span. obispo portug. bispo. - $g$ : span. esparrago (innl. a) portug. spargo; ercir cojer, wie in andern Sprachen. $d$ : zunächst natürlich auch hier verde frio lerdo sueldo caldo; dann neto puto mit Synkope vor ${ }^{1}$ raudo lauda (Baist, Zeitschr. V 245)

1 Sie könnten auch aus ned(e)do pud(i)do, mit Übergang von gedehnter Media zu einfacher Tenuis gebildet sein. 
jaudo nach der Lautabstufung, cuenda mit gegenseitiger Assimilation; da aber bei -mpid- aus -mpit- dies nicht eintritt und in letzterem Falle die Synkope nicht wohl vor die Lautabstufung gesetzt werden darf, so sind die $Z$ wischenglieder zwischen comped-a und cuenda nicht $m p d$ mbd nd sondern mbed- mbd; pardo. Wegen lindo vgl. S. 216. Daneben tritt Ausfall des $d$ ein in tivio turbio pudio escalio lacio lucio rancio recio limpio sucio livio mustio. Nicht alle diese Wörter tragen echt volkstümliches Gepräge, aber auch nicht alle dürfen als halbgelehrt bezeichnet werden. $\mathrm{Da}$ wo mehrfache Konsonanz die tonlose Silbe anlautet, ist die Sache klar: aus physiologischen Gründen mulste der Vokal bleiben, ${ }_{-} d \underline{v}$ fiel. Aber weshalb tivio neben lauda? Ich weifs nur den einen Ausweg, dafs der Ausfall des Nachtonvokals und des intervokalischen $d$ zu gleicher Zeit begann, so dals da, wo beide Bedingungen vorlagen, bald das eine bald das andere zuerst stattfand. - Nebeneinander stehen bravio und bravo, über letzteres vgl. S. 219; bravio erklärt Storm a. a. O. aus *brávio wie rocio aus * rócio roscidus: in beiden wird man eher mit Diez, Gr. II 365 Weiterbildungen mit rom. io sehen. Abfall der ganzen Endung konstatiert Baist, Rom. Forsch. I I I I in befo bif żdus; sehr zweifelhaft ist cerdo sordidus; tolva (Mühltrichter) nach C. Michaelis $27 \mathrm{I}^{2} \S 37=$ turbida passt weder lautlich noch begrifflich: tubula genügte dem Laute, der Begriff und die Nebenform torva weisen auf turbo, inis Kreisel (vgl. span. tolvanera Windsbraut), allein die Proportion * turba: turbo :: ital. dazia : datio wäre aus mehr als einem 'Gruude verfehlt. Ich erwähne noch marchito, was auf marcho aus marcidus hinweist. Im Portug. findet sich: tivio, dann, im Gegensatz zum Span. Ital. Prov. Frz. Rät., aber in Übereinstimmung mit dem rum. neteo, ferner ranço neben murcho, endlich rijo aus rigidus, oder vielleicht aus ridigus? - $b$ : span. cañamo zeigt Assimilation, portug. caco = cacabus, gal. Santiago Vokalisation oder Ausfall des $b$. Span. orespe $=$ orezbe $=$ orecibem statt oreficem (aurifex.). - n: aginem -en, iginem -in: es frägt sich, ob die Zwischenstufe $\operatorname{agn}(\mathrm{em})$ oder ain $(\mathrm{em})$ sei, eine Entscheidung ist kaum möglich: aus a $(g)$ inem könnte en entstehen wie aus laico lego; aus ainem wie aus faitum hecho, aus disdignum desden; der Mangel des auslautenden - e kann kaum gegen die erste Möglichkeit sprechen. -minem wird zu mne mre mbre, die Beispiele sind zu bekannt, als dals ich sie anzuführen brauchte. Auch in andern Fällen, wo $n$ auf Konsonanten stölst, wird es $\mathrm{zu} r$ : landre ${ }^{1}$ liendre sangre, selten $l$ : engle; geht schon ein $r$ vorher, so wird das zweite abgeworfen: carpe garma baluma grama. Daneben nun arcén (arger bei Priscian 35) cercén mit secundärem Accente: es scheint, dafs nur an vorhergehende Explosivlaute und $m$ das $n$ sich so enge anschlors, um $n$ und dann $r$ zuwerden, dals es bei Zischlauten sich ferner hielt und so die volle Silbe en erhalten blieb. Virgen margen origen imagen erweisen sich durch $g$ als Latinismen (das erste und letzte sind Worte der Kirche): joven ist

1 Danach ist Ascoli, Arch. IV 399 Anm. I zu berichtigen. 
wohl durch alte Ableitungen wie jovenil jovenica u. a., orden durch ordenar gehalten; endlich noch peine aus pectine(m), woraus pelne (vgl. petral * pectrále) und mit Übergang von tn in $k n$ (vgl. norm. anqu'nee neben ant'nee lit. sekmas aus septmas). Schwierig ist empeine nebst empeña empella = lat. impetigine $(m)$ portug. empigem, auffällig in beiden Sprachen ist der Mangel des $v t^{v}$, legen wir *impiginem zu grunde, so sollte das Wort *empin lauten; die erste der mit Femininendung versehenen Formen (die zweite dürfte eine dialektische Variation davon sein) entspräche dagegen gut einem empig(i)n+a; empeine liefse sich etwa erklären: aus impetigo, iginem entstand durch Umstellung der Laute impegito impegitne $(m)$, aus letzterm entweder mit Ausfall des $i$ impectne $(m)$ oder des $g$ impeitne $(m)$; da portug. Form aber erklärt sich so nicht. Was endlich die "gelehrte" Form empige betrifft, so ist sie offenbar eine falsche Rückbildung. Ganz unwahrscheinlich ist die Herleitung von légamo aus uligo. Anders als $n e(m)$ wird $n u(m)$ behandelt: hier tritt nur bei anlautend $s$ und bei dentalem Verschlufslaut Synkope ein. cuebano (coffre aus dem Frz.) huergano huerfano tabano sabano (trepano) rabano pampano tempano (limbre frz); rienda ; asno fresno lesna limosna hanzo rezno cisne; jalde entspricht ital. giallo, und erklärt sich wie dieses, zu $l d=\|$ vgl. espalda celda. In dueño laña ist die Synkope lateinisch. Nicht klar ist laúna laganum; Auflösung von Gutturalen in $u$ statt $i$ kommt sonst im Span. nicht vor.

Im Portugiesischen liegt die Sache etwas anders, da hier einerseits $g$ vor $e i$ nicht ausfällt wie im Span., sondern zu $\check{z}$ wird, andrerseits intervokalisches $n$ fällt. Wir haben daher:-agine(m) agem, nebst empigem aus *impedigem, letzteres etwa einer alten volksetymologischen Anlehnung des lat. impetigo an impedicare zu verdanken; -inem em: homem origem nubem margem jovem; -ina aber -ea: femea codea lendea landea redea gemeo or fáo orgao rabão (tavádo), steht aber $s$ im Anlaut, so fällt der Vokal zuerst: asno esmola (aus * lemosna) durazne cisne, sogar cerne. Auffällig ist der Mangel des $m$ in carpe, vgl. jedoch die gleich zu nennnenden pente und trempe, und bage neben bagem. Trempe ist aus trepinem trepnem, pente aus pectinem pectnem entstanden. D' Ovidio, Gramm. port. S. I I und 56 sieht in pente gal. peinte Nominativformen, allein das Gallizische besagt überhaupt nichts, da auch $-n$ in sekundärem Auslaute fällt; wenn neben pente noch pentem vorkommt, so beweist das nichts gegen die Neutrum S. 79 gegebene Erklärung: auch wenn, wie ich annehme, der inlautende Nasal dem auslautenden seine Entstehung, verdankt, so bedingt das keineswegs sofortigen Untergang deś letztern; wenn die Nasalierung in pente allenfalls aus ct erklärt werden kann (vgl. span. enteco), so ist dies bei dem ganz parallelen trempe nicht möglich; nach meiner Auffassung ist das $n$ vom Subst. ins Verbum gedrungen (pentear statt peitear) pentelho erklärt sich eher aus *pectniculum, als aus pentem +elho. - cofre und timbre stammen wie die entsprechenden spanischen Worte aus Frankreich, ferra neben ferragem entspricht ital. ferrana S. 21 8; calina, das auch 
im Span. Prov. Norm. erscheint, und sarta sind weniger klar. Span. calina könnte calinta sein, auch die norm. Form, nicht die prov. und portug. fügen sich dieser Erklärung. Setzen wir calina (das von calere geformt ist, wie ruina von ruere, agina von agere u. s. w.), so ist für drei Gebiete die Frage gelöst, im Portug. aber erwarten wir calinha, doch zeigen noch einige andere Wörter -ina statt inha, vielleicht unter span. Einflusse. In sarta scheint die schon einige male bemerkte Vertauschung von ago mit ana vorzuliegen. $-m$ : bei anlautend einfacher Konsonanz synkopieren beide Sprachen: span. portug. alma span. yermo portug. ermo, span. mismo portug. mesmo, span. maresma; span. bisma und bilma, diezmo und delmo, span. portug. blasma aber lagrima. Wegen alamo vgl. Baist, Rom. Forsch. I I 32. Die span. Dialektformen mit $l$ verhalten sich zu den gewöhnlichen mit $z$ (d. i. ठ), wie -algo (=aticum) zu -azgo; wenn Baist a. a. O. S. I 37 decuma für delma ansetzt, so beruht das wohl auf einem Druckfehler. - $s$ kenne ich nur in zwei griechischen Wörtern: codeso aber saja aus *jasa (dıai - Auch bei $r$ bleibt der Vokal selten. Ich sehe von den Infinitven, die eine eingehendere, aber specifisch morphologische Untersuchung verdienten, ab. Bei $n^{\prime} r$ tritt im Span. Metathesis ein: yerno viernes tierno, das Portug. schwankt terno aber genro; m'r schaltet ein vermittelndes $b$ ein: ombra, cogombro cambra; dr: yedra; pr: licbre; ist aber der tonlose Vokal $a$, oder stehen im Anlaut zwei Konsonanten, so hält sich der Vokal: gambaro camara cantaro farfara; arbol.

Über die Qualität des Vokales ist wenig zu sagen: wo er bleibt, ist er $e$, im Span. wird $\operatorname{mu}(m)$ zu. ano, ob auf lautlichem Wege (vgl. das Ital.) oder weil mehrere der zugehörigen Wörter von hause aus, bezw. durch Assimilation schon ein $a$ hatten, lälst sich nicht mit Gewirsheit sagen. Auch das ebengenannte camara kann sein $a$ durch Assimilation an dasjenige des Stammes oder an das $r$ erhalten haben. Das erstere ist wahrscheinlicher.

15. Provenzalisch. Hier gehen die Dialekte verschiedene Wege: die einen folgen dem spanischen die andern dem französischen Beispiele. Im allgemeinen tritt die Synkope ein, so oft es die umgebenden Laute gestatten, und zwar meist nach der Lautabstufung, wodurch das Prov. scharf vom Frz. geschieden wird. Wie im Span. so scheint auch hier in einzelnen Dialekten ${ }^{\prime}$ stets tonlos zu sein: wenigstens wüfste ich Schreibungen wie $t z$ kaum anders $\mathrm{zu}$ erklären, daneben $\operatorname{tg} g c z$. Leider stand mir nur das Wörterbuch von Azais, in welchem derartigen dialektischen Unterschieden nicht die gehörige Aufmerksamkeit geschenkt wird, zu gebote. Ich kann daher im folgenden nur Andeutungen geben: die weitern Fortschritte der provenzalischen Dialektforschung müssen die genauere Grenze zwischen spanischem und französischem Systeme finden lassen. - ex icis: pouce, aber pieuze euze feuze sauzc rounze selse; pansi; erso (cast.); dagegen kann gasc. saligo bearn. saligue salíca sein, sofern wenigstens der Ton auf dem $i$ steht; erpi erpe arpe (1.) drpio erpio arpeie (d.) drpego arpegne (bord.) arpei (d.) erpi 
(Burset) gehen auf hirpic-um hirpic-am zurück; roumi auf rumicum. Zehnerzahlen: douze (l. nizz.) doutze (b.) doutge (g.) dutze (auv.), bei andern sind die Formen noch zahlreicher. zcus, a: tourjo tourgo neben tourigo, in Rouergat (nach Aymeric) margo cargo deminge, -age, piege nă̌o pergo sieğe feğe canounge und canourgo; clergue; aber toul. dimentge tönend oder tonlos? d. dimenche a. doumenche; farjo zeigt überall tönenden Zischlaut, nur b. hourquio; fulica : fauco. Neben synkopiertem mourgo wird mouneco angeführt, wohl gask., wenigstens soll manego (neben margo mancho) gask. sein. Abfall der Endung bei anlautend mehrfacher Konsonanz zeigen ruste und sendi, letzteres natürlich nicht rein volkstümlich; Accentverschiebung tritt ein in senéco, die linke Hand, das wohl nicht auf ein heteroklitisches *senéc- zurückgeht, sondern aus sénego mit Accentverschiebung gebildet ist (vgl. unten), und junego neben junja. Verba auf icare penjar pastorj. plonj. veng. jutg. autorj. cavalj, selbst domesj., aber repropchar (reprobic.) clopch. Das erstere ist auffällig, wie soll bäc zu pch werden, wenn die Synkope bei den übrigen erst stattfand als man schon big sprach? Daher ist vielleicht doich das Diezsche repropiare vorzuziehen.

ficatum ist hier nur als * fiticum erhalten: fege fugi (m.) fetge feche (l.) hetge hitge (g.) hitye (b.). - - t. Hier macht es sich besonders geltend, dals mir genaue Angaben über die einzelnen Dialekte nicht zu Gebote stehen; die Formen mit tönendem und tonlosem Verschlufslaut gehen sehr häufig neben einander. habitus maldute, debitum deute, nied. lim. deude, quer. dioute; digitus det, cev. de gask. dit dauph. dey.; cubilus: couide coucide (m.) coupte (bord.) couive (l.) couve (n.); cucurbita coucourdo und courdo (vgl. S. 226 Anm. I) daneben * cucurbica couje.; subitus subte und sutte (volkstümlich?) domitus dounde, aber verb. doundd und dountd. sabatum dissate nied. lim. dissade gasc. dichatte prov. dissato; gabatum apr. gauda (von Raynouard Lex. Rom. II 40 fälschlich lagauda gelesen), gauto gato gaudo, auch gavido, letzteres prov. - computare countd und coundd. - Für den Dialekt von Rouergat erhalten wir durch Aymerics Darstellung ein etwas einheitlicheres Bild (A. achtet auf diesen Punkt natürlich nicht). Die Synkope ist da vor der Lautabstufung eingetreten: dissáles bontd (vanitare) gauto tonto (amita) dutd, cunte molaute; in deude hat gegenseitige Assimilation stattgefunden: $b-t \quad z u d$; in dundd ist vielleicht der Innlaut dem Anlaut angeglichen; placitáre ploiğa, cogitare cuiğa. Die Verbalabstrakta der Verba auf nd schwanken zwischen nd und nt: pento rento atento neben atendo. doch ist hier bekanntlich das Schwanken nicht phonologischer Natur: die Formen auf $-d$ gehen vom Präsens, diejenigen auf $-t$ vom Participium aus. Endlich sind noch die Reflexe von tarmes ztis zu nennen, die im Prov. unter sehr verschiedenen Gestalten erscheinen: arto ardo, deren Anlaut durch Dissimilation verloren ging; daneben arno darno (lim.) argno (rhod.) arlo (gasc.), die alle auf $t$-armula zurückgehen können, und sich genau mit ital. tarmola decken (zu rl rn vgl. frz. marne poterne prov. marno) - apr. coesque. 
- d: lourd, -o; fre frech u. s. w.; fade verd rai f. -de (cev. rapidus?) net, $o$; sonst bleibt $i d, \underline{v} d^{v}$ muls fallen, im Fem. ist -ezo zu erwarten, was sich im Apr. richtig findet. Die neue Sprache gleicht nach beiden Seiten hin aus: bald folgt das Fem. dem Mask., bald umgekehrt. 1. cande, cando, aisse, $o$; yuite, $o$; rance, $o$; rege, $o$ (neben rede, 0 , bearn. rette); horre, $o$; tebi, o (weshalb nicht tebe? Dialektische Verschiedenheit?); 2. coubès, eso, tebès, eso. Ich erwähne noch die Neubildung moude, o (mütidus). bycida zeigt durchweg Assimilation des $d$ an das $s$ : bouito bouisto boueito (m.) boueitio (lim.) u. s. w. $n$ : aginem age, was wohl in folge der Kollision mit age aticum zuweilen $m$. wird: plantage $m$.; daneben proubajo aubujo caluge, und mit uginem statt -udinem eiruge. Über calins vgl. oben S. 230. Alte Feminina behalten natürlich $n$ und synkopieren: alesno almosno; wenn pagina pajo lautet, so ist das wohl eine Entlehnung aus dem Frz., femina synkopiert ebenfalls, dabei wird $m^{\prime} n$ teils zu $n$ (nied. lim. querç. g. auv.) teils zu $m$ (rhod. a. niç m. g.), teils bleibt es unverändert (d. g.). Nur im Nexus tina bleibt der tonlose Vokal, der Accent wird aber vorgeschoben (vgl. unten) coudeno aseno. Für *bodina finde ich folgende Angaben: bouino boueino (lim. d.) boueno (for. d.) boino (g.) borno (b.) bouorno (rouerg.): die zwei letzteren mit Ausfall des Vokals, die andern des Konsonanten. Bei den Mask. herrschen die $n$-losen Formen vor: fraisse ase casse engue pampo (aus pampanus!); daneben engle pampol; endlich gasc. aine for. agne lim. d. ane. pecten erscheint als pegne pigno penche, die alle auf pectne zurückgehen; juvenis teils als joune (nied. lim.) teils als jouve. Auch incudinem zeigt eine wahre Musterkarte von Formen: enclume enclum (a. nizz.) enclumi (g. m.) mit Anlehnung von udine(m) an umen; enclugi (g. m.) encluge (nizz., rouerg.) enclutge Vertauschung von udine(m) mit ugine(m), endlich enclusi (l. g.) encusi (nied. lim.) stellt -udine(m) dar. - $m$ : baume balme (balsamum) blame deme (décimus) amo bearn. anme quer. armo (anima) erm (éremus). - -ru(m), -re(m) stets zu $r$, das sich nach Konsonant als -er, nach Vokal als re darstellt: naisser - moure. Wichtig sind faire dire aus fakre dikre: die Synkope ist älter in diesem Falle als die Assibilation. Wir ersehen daraus, dafs zuerst $r$ sich an einen vorhergehenden einfachen Konsonanten anschlols, und zwar schon in sehr früher Zeit.1

Ein Wort über das Verhältnis zum Auslautgesetz ist hier zu sagen: die Synkope ist durchaus jünger, Wörter mit einer Silbe nach dem Tone, werfen den Vokal (aufser $a$ ) ab, solche mit zweien behalten ihn als $e$; wäre hab'tus vor dem Auslautgesetze entstanden, so mürsten wir im Prov. mal-aut, nicht -aute erhalten; vgl. das Frz.

Aus dem Katal. will ich nur das eine anführen, dals -mð̌t- zu

1 Was Horning lat. c S. 37 sagt, ist unrichtig, vgl. unten; wichtig sind prov. claure creire, die nur auf altes claudre credre, nicht auf claud(e)re cred(e)re zurückgehen; cred(e)re gäbe crezer creder, die Bartsch, Chrest. S. 437 zwar anführt, die er aber unter Verkennung der prov. Laut- und Flexionsgesetze selbst gemacht hat. 
$n n$ wird, d. h., dafs die Synkope nach der Lautabstufung, aber vor der Assimilation von altem $n d$ zu $n n$ stattfand; dafs $n d$, nicht $n t$ das Mittelglied ist, ergiebt sich daraus, dafs altes $n t$ bleibt. Daher fenna femita. Einiges andere nachher.

I6. Das Französische macht weniger Schwierigkeiten; die Dialekte kommen kaum in Betracht, da sie, soweit ich sie übersehe, keine wesentlichen Abweichungen von der Schriftsprache zeigen. Im allgemeinen lälst sich als Regel aufstellen: die Synkope ist älter ais die Lautabstufung und als der Übergang von $a$ in offener Silbe in $e$, aber jünger als das Auslautgesetz.

$c$ : puce pouce fọce ponce ronce panse herse verse (vertex?) lotr. burg. sausse. code gehört der einst lateinischen Gerichtssprache an. juge ist entweder judic-um oder postverbal, souris folgt, vom Nom. aus, dem Beispiele von vervex. meleze aus mellarix $\mathrm{Wb} .638$ ist höchst unwahrscheinlich. Auffällig ist gegenüber dem tonlosen $s$ in pouce puce das tönende von yeuse; Horning, lat. C S. $16 \mathrm{f}$. erwähnt das Wort nicht; es kann Anlehnung an -euse osus vorliegen. douze u. S. w. mit Assimilation $d c^{\prime}$ zu z. Bei $c a$ co sind verschiedene Fälle zu unterscheiden. Der Hauptregel fügen sich: dimanche manche nache rache peche; in piege hat gegenseitige Assimilation stattgefunden; in forge die Konsonantenkombination im Auslaut der Silbe die Synkope bis nach Eintritt der Lautabstufung und damit verbundener Auflösung des Nexus $b r$ in $u r$ aufgehalten. Daher ist vielleicht perche nicht per $(t) c a$ sondern pertga. Serge und barge kommen aus dem Süden (prov.). Anders liegt die Sache bei -icus: aticus giebt age vgl. Ascoli, Arch. I 77 Anm., sedicum (so soll es in meinem Neutr. S. 155, nicht sedium heifsen) siege; allerdings findet sich Afrz. neben nache auch nage (Berthe au gr. p.). doch darf man daraus nicht auf ein Schwanken zwischen tönendem und tonlosem Konsonanten überhaupt schliefsen: entweder liegt eine specifisch wallonische lautliche Abweichung, oder eher eine Angleichung an die zahlreichen Subst. auf -age vor. Daraus ergiebt sich also, dals _ica sein $i$ früher verlor als Licum, was wohl mit dem auf $a$ ruhenden Nebentone zusammenhängt. Die Verba auf zcare zeigen doppelte Formen: vor dem Tone tönenden, nach dem Tone tonlosen Palatal; das alte Verhältnis zeigt sich noch heute in revanche - venger vengeance. Es kann sich fragen, ob daraus ein Anhaltspunkt für die Geschichte der Synkope zu gewinnen sei, ob man also in einer bestimmten Periode vin(d)cat - vind $(c)$ gáre sprach, oder aber ob vin(d)cat vin(d)cáre erst nach Eintreten der Synkope zu -vanche venger umgestaltet wurden; für letzteres liefse sich etwa fourche - afrz. fourgier anführen, doch verliert das Beispiel jede Beweiskraft, wenn wir für das Verbum statt furcare ein nach dem Muster der Verba auf -icare gebildetes * furicare (it. furicare venez. furegare sard. forogai) zu grunde legen, für ersteres sprechen delgié berger und bei schliefsendem Dental landier, das ich nicht mit Diez von dem dunkeln mlat. andena herleite, sondern für eine Ableitung von ames amitis halte. Sonst haben wir also: arracher clocher coucher 
Epancher facher nicher oscher pancher plonchier (afrz.) prêcher neben charger enferger mänger plonger ronger afrz. targier venger. - Aus der Betonung źco icare entstand oi - oizer (vgl. fois - foi-iee); daneben steht pri (pręco) - proi-ier was zunächst ploi (plico), ploi-ier (plicare), dann mehrere der Verba auf oyer in Verwirrung brachte, daher die Formen auf -ier. Beispiele Diez, Gr. II 399. Es bleiben noch grammaire (Tobler, Rom. II 24I-244) artumaire (G. Paris, Rom. VI I 32) daumaire (Tobler, K. Z. XXIII 4 I 4 ff.) firie

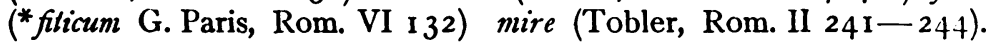
Das $r$ ist aus $\delta$, dieses aus $d t$ entstanden (Havet, Rom. VI 254), wir haben somit neben $\stackrel{\bullet}{g} e=\underline{v}$ ticum in einigen Fällen $\stackrel{v}{d i c}=$ - ticum: die Synkope ist erst eingetreten, als $d$ schon zu $\partial$ geworden war; während also bei den alten Wörtern das $d$ beim Zusammentreffen mit $i$ noch verschlufslaut war, so dafs di zu $\breve{g}$ werden konnte, ist es hier schon halb spirantisch, so dafs es leicht in den verwandten Linguallaut hinübergleitet. Die spätere Synkope erklärt sich bei fétácum (diese Form ist zu Grunde zu legen) daraus, dafs das vollere $a$, nicht das leichte $i$ der tonlose Vokal war; die übrigen alle sind halbgelehrte Wörter. Ganz wie mit firie verhält es sich mit monachus moine und * canonachus canoine, das a hinderte die Synkope für einige Zeit.

Dagegen ist nfrz. foie = fécatum, zu dem es sich ganz so verhält wie digilus $\mathrm{zu} d o i(g t)$, nur mufste tonloses $a \mathrm{zu} e$ werden, nicht verschwinden. (Dafs $a$ hier $e$, oben $i$ ergiebt, erklärt sich aus dem nachfolgenden Konsonanten.)

$-t$ : jante, tante 1 friente fiente afrz. sente (noch heute im Norm., davon, nicht von * semitarium sent-ier) cointe doute dette imeute ${ }^{2}$ rente u. s. w. (Arch. Gl. VII 140), voute hôte vanter hanter conter tentiv peter reter convoiter (cupiditare streng nach den Lautgesetzen; Schelers Zweifel bei Diez, Wb. 717 entbehren jedes Grundes) jatte (aber jadeau vor dem Tone; Diez kennt norm. jade, was entweder aus dem Dim. zurückgebildet oder zu erklären ist, wie coude. Joret verzeichnet gate), daneben joue-gaula. brt schwankt übrigens, es kann gegenseitige Assimilation stattinden: coude gourde (neben courge) malade bondir. - Daneben stehen nun doi $(g t) \mathrm{Pl}$. doie, plait (vgl. plaidier) afrz. vuit Fem. vuide (nfrz. vide vom Fem. auch ins Mask. gedrungen), cuidier, ferner fuite pleite exploitier ruit. Während fuite u. a. aus einem nach dem Muster von ductus u. s. w. gebildeten fucta entstanden sein kann, widerstreben plait und vuit vocitum dieser Annahme, leizteres weil es nicht mehr Part. sondern Adj. ist, beide wegen der zugehörigen Formen mit $d$. Die Sache verwickelt sich noch, weil beide bei Eintritt des Auslautgesetzes zweisilbig gewesen sein müssen. $\mathrm{Ob}$ das $-t$ nur in folge seiner Stellung im Auslaute tonlos war, liefse sich noch fragen. Die grölsere Wahrscheinlichkeit

1 Die beste Erklärung des $t$ - bei Canello, Arch. Gl. III 34I Anm.; er hätte auf neap. vavo $=a v o$ hinweisen können; vgl. oben S. 221 Anm. I, 222 und nyanya (amita) in Ampezzo.

2 movita, nicht mota; daher trémousser $=$ transmov(i)tiare. 
spricht dagegen. Zwar scheint die Annahme das $d$ in plaidier gehöre nur endungsbetonten Formen an, zu scheitern an vide. Und doch ist das der beste Ausweg: vide kann unter verschiedenem Einflufs z. B. dem des Verbums, oder anderer Adj. (vuit : vuide : : vert : verde) stehen; dites (dicitis) faites (facitis) ${ }^{1}$ können, so viel ich sehe, nach keiner andern Form aus * dides faides umgebildet sein. Die Erklärung dürfte dieselbe sein, wie bei den entsprechenden ital. Formen, es bleibt nur die Frage: weshalb giebt facitis faites aber placilu $(m)$ plait? Hatten beide Worte vor dem Auslautgesetz zweisilbige Formen, und vermochte nun das $i$-Element der palatalen Konsonantenverbindung den $i$-Vokal des Auslautes zu dehnen (vgl. got. und lit. $-i s=j i s)$, so dafs es widerstandsfähiger war? Der Hinweis auf nuit könnte nicht dagegen sprechen. Ich schliefse bier gleich noch ein anderes an. Horning, Lat. C $37 \mathrm{ff}$. erklärt faire aus faisre wie veintre aus veinstre vençre. (Der erste, der diese Erklärung giebt, ist weder $\mathrm{H}$. noch Mussafia, Litbl. 1883 Sp. 279 sondern schon I 874 Ascoli, Arch. II I 19 Anm. I für tordre.) Allein $\mathrm{H}$. beachtet nicht, dafs bei anlautend mehrfacher Konsonanz die Synkope, wenn sie überhaupt eintritt, später erscheint, als bei einfacher, dafs sie also dort sehr wohl jünger hier älter sein kann als die Palatalisierung, ebenso, dafs ein $r$ vermöge seiner physiologischen Beschaffenheit leichter als jeder andere Konsonant sich hart an vorhergehende Explosivlaute anschliefst. Ohne auf die verschiedenen Unwahrscheinlichkeiten, die sonst der Entstehung von faire aus faisre anhaften, aufmerksam zu machen, will ich nur auf das sehr instruktive Verhältnis im Prov. (S. 232) hinweisen, und auf katal. fer pler (= frz. plaire, nicht plaer wie Mussafia, 7 Meister $\S 57$ meint; mahel giebt nicht mel), dir dur, neben denen keine Formen mit $u$ existieren, die doch notwendig da sein mülsten, wenn $c^{\prime} r$ vorläge. Weiteres über die Verbalformen und namentlich über H.s Bemerkung über fait - plaist hat mit der Behandlung tonloser Pänultima nichts $\mathrm{zu}$ thun. In digita mufs die vollständige Verfüchtigung von $\because g$ v vor die Synkope und das Auslautgesetz fallen. - p: coup; tvique jenes vor, dieses nach dem Auslautgesetze. - $g$ : asperge ist zur Zeit, da man in Paris ar wie er sprach, wohl aus Italien nach Frankreich gekommen; afrz. viautre *velirum. zdus: verd froid afrz. roit chaud net soud lourd alle mit alter Synkope; rade tiede fade sade mit Synkope nach Eintritt des Auslautgesetzes, vor Übergang des $a$ in $e$, vor Ausfall von $\underline{v} d \underline{v}$, zu einer Zeit, da $\bar{e}$ in Position noch diphthongisch war, oder wenigstens nach Auflösung derselben werden konnte. Der anlautende Konsonant assimilierte sich dem $d, d d$ wurde durch die Lautabstufung $\mathrm{zu} d$. In boîte und moiste assimilierte sich das $d$ dem tonlosen $s$. Ich erwähne noch norm. $m \varepsilon$ (M.) magide $(m)$, hier ist die Synkope jünger, da $g d$ das a geschützt hätte; der Grund könnte derselbe sein, wie im Ital.

1 Danach faimes statt faismes, das, wie Horning lat. $c$ S. 39 richtig gesehen hat, facimus entspräche, seine Erklärung überzeugt mich nicht; 
(S. 216), doch hätte dann $a+i a i$, später ouai ergeben; daher in magide wohl das $g$ und $i$ ebenso spurlos verschwunden sind, wie in digitus. Joret hat neben me reide; hätte er eine rationelle Orthographie angewandt, so mülste er reid schreiben. Im Nfrz. ist $e$ in raide vom Fem. aus übertragen, stütz-e kann es nicht sein; dafs roid nicht auf rig(z)dus sondern auf rĭgdus geht, lehrt digitus. Mehrfache Konsonanz hindert die Synkope, 드 mufs natürlich fallen: rance pâle. Wenn épave = expavidus ist, so muls es später aufgenommen sein, da $a v$ zwar zu $o$, vielleicht zu $a$ werden, auf keinen Fall aber bleiben kann. - $b$ : chanvre mit unorganischem $r$; Jacobus mit Übergang von $b$ in $m$ Jaime.

$n$ : âne aumône frêne rêne aisne. Schwieriger ist chêne. Die von Diez vorgebrachte und zuletzt selbst von Horning wiederholte Herleitung von quercinus ist lautlich ganz unstatthaft. Prov. casse weist auf * cassinus, das frz. chasne gäbe: so lautet die afrz. Form z. B. bei Chrestien, während dem nfrz. chêne eher ein *caxinus gerecht würde. Das Wort mag ein altheimisches sein, etwa keltisch; man könnte an osk. cas-nar "der Greis" denken, da die Eiche sehr alt wird, doch ist casnar zunächst „der Weifse“, so dafs eher, wenn diese Wurzel kas zu grunde liegt, vielleicht einst die Weirspappel damit bezeichnet wurde: Übertragungen von Baumnamen sind häufig (vgl.

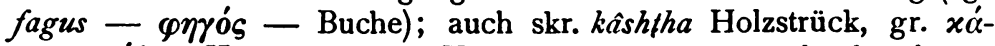

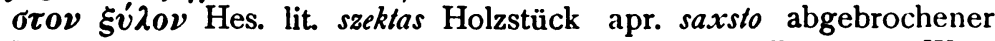
Baumstamm gehen auf ein kaks zurück; dafs das allgemeine Wort für Holz die Eiche speciell bedeutet, kommt auch sonst vor. Allein beweisen läfst sich die Sache nicht. Will man zugeben, dafs das frz. prov. Wort für „Eiche“ auf einem Nebenflüfschen in den Hauptstrom eingeflossen sei, und bedenkt man, dals idg. das wahrscheinlichste ist (sofern das Baskische nicht aushilft; sonst wohnten vor und neben Celten die indogermanischen Ligurer in Frankreich), so läfst sich auch die Berechtigung nicht abstreiten, in den verschiedenen idg. Sprachen, auch wenn sie noch so ferne liegen, nach einem lautlich und begrifflich entsprechenden Worte zu suchen. Freilich bieten sich in diesem Falle gewöhnlich mehrere Möglichkeiten, zwischen denen schwer zu entscheiden ist. - rine; terne auronne plane Etienne antienne (antëphona) orne (carne aus dem Prov. entlehnt? vgl. aber charn-iere) jeune aginem ain, iginem in: die Synkope ist älter als die Palatalisierung und das Auslautgesetz, agne $(m)$ wird wie altes $g n u m$ behandelt. Die Beispiele sind bekannt; ich erwähne nur norm. craqu'lin ganz regelmärsig aus cartilaginem gebildet; Joret führt es ohne Not auf eine Wurzel crac zurück. Geht dem $g$ ein Vokal voraus, so unterbleibt die Synkope, das $-n$ fällt verhältnilsmälsig spät; vierge marge orgue; vertige und image sind gelehrt. femme afrz. auch fenne in Poitou (Görlich S. 80) und Normandie (Faulde, Zeitschr. IV 56r), worin $m^{\prime} n$ wie im Prov. behandelt wird. lerme larme homme - don $=$ domnum; aber farcin nicht mit Scheler farciminum sondern farcimen oder, da -go bei Krankheitsnamen nicht selten ist, farcigo. 
In mehreren Fällen wird $n$ zu $r$ ordre coffre diacre pampre timbre, sehen wir von dem der Kirchensprache angehörigen diacre ab, so haben wir im Anlaut aufser bei coffre mehrfache Konsoñanz, im Auslaut aufser bei ordre $-u$. Zur Zeit, da die Synkope eintrat, war also wohl $-u$ schon gefallen, $-e$ noch nicht, $-n e$ blieb, $n$ wurde $r$, ordre neben orne ist vielleicht später aufgenommen; charme $=$ carpinu $(m)$ ist nur eine scheinbare Ausnahme, es ist von carpinem (vgl. die ital. span. portug. Form) auszugehen, woraus durch gegenseitige Assimilation (labialer Verschlurslaute, dentaler Nasal = labialer Nasal) charme entstand. - * cicinus cigne: hier ist die Synkope zwar vor dem Eintritt der Assibilation, aber nach dem Auslautgesetz eingetreten. Wie aus cic'nus cigne so aus pectnem peigne, nachdem $t$, als der mittlere von drei Konsonanten ausgefallen war (anders D'Ovidio, Gram. port. IO Anm. 3). Auffällig ist beim zweiten Worte der Vokal; nach dem von Horning lat. C S. 2 I f. richtig formulierten Gesetze sollten wir pigne erwarten, da ital. pęltine auf \& weist; doch wird "singuläres singulär behandelt"; die schwere Gruppe -cln hat vielleicht die Entwickelung von $\xi \mathrm{zu}$ ie gehindert. - Aus galbnus entsteht gal(b)nus jaune, aus germ. *alina aune. Hier ist noch aune = lat. alnus $\mathrm{zu}$ erwähnen. Im Lat. wird altes ln zu $l l$, nur wo die beiden Konsonanten verhältnismärsig spät zusammenstofsen, werden sie geduldet (alnus $=$ alsnus, vulnus = vulsnus, balneum $=\beta \alpha \lambda \alpha \nu \varepsilon l o \nu)$. Im Rom. sucht man sich auf verschiedene Weise ihrer zu cntledigen: in balneum geht $l$ spurlos verloren, in alnus ebenfalls in rät. und rum. Dialekten; fürs Span. vgl. Diez II ${ }^{\text {b }}$ alama und Baist, Rom. Forsch. I frz. aume muls wohl alinus mit Svarabhakti sein: Stütz-e (ein bequemer Begriff, der etwas schärfer definiert werden sollte) kann das $e$ nicht sein, da Irn auch keines zeigt. - juvenis zeigt im Afrz. noch einige beachtenswerte Nebenformen: $v$ kann vor dem $n$ tonlos werden juefne, $n$ wie in den andern Fällen zu $r$ werden juefre; oder $v$ bleibt tönend, $n$ wird $r, l, v$ geht im vorhergehenden Vokale auf: joule (wallonisch, vgl. Settegast, Jean de Thuim XXI), oder endlich on assimilieren sich jenne (häufig im Doon de Mayence).

$m$ : âme blâme erme chaume larme carême dîme centimel -ilme.

Über $-r$ ist nichts mehr zu sagen: dafs Wichtigste habe ich oben bemerkt; $m^{\prime} r n^{\prime} r$ s'r lassen den homorganen Explosivlaut zwischen die zwei Dauerlaute treten. Weitere dialektische Behandlungen des Nexus s'r haben mit der Synkope nichts zu thun.

Doppelkonsonanz im Auslaut der Nachtonsilbe ist im Lat. nur möglich, wenn der zweite Konsonant ein $r$ ist. Der Vokal sollte $e$ sein, und ist es auch in tenebrae integrum porcetra penetro u. a., daneben aber findet sich auch $i$ in tonitrus, wohl unter Einflufs von tonitus. Im Rom. wird der Accent vorgeschoben, der Vokal als Kürze behandelt, vgl. Diez, Gr. I 403, Ascoli, Arch. I 18 Anm. I.

1 Der Vokal von dîme aus übertragen, ähnliches im Ital.: D'Ovidio, Giornale di fil. rom. I 69, wo auch auf die frz. Formen hingewiesen wird. 
Die Ausnahmen erklären sich teils durch lateinischen Einflufs (ital. tenibre), teils durch alte Umstellung des $r$ (span. estruendo ${ }^{1}$ ). Ital. pollędro pullı̆trum (Ascoli a.a.O., von Scheler bei Diez 727 ganz verkehrt wiedergegeben), span. portug. potro frz. poutre weisen auf alte Flexion púlliter pullétrum, im Span. Portug. Frz. ist die Synkope älter als der Untergang der Deklination. Im Ital. drang der Vokal des Nom. auch in den Acc. - Neben cúlczila ist cuilcilra schon alt; das erste Wort scheint die Accentuation des letztern beeinflufst zu haben, daher ital. coltrice mit Metathesis, um ein gangbares Suffix zu erhalten; mit regelmälsiger Accentuation span. colcedra portug. cozodra. Sonst synkopierte Formen, teils mit, teils ohne $r$ : span. portug. colcha (das portug. Wort aus dem Span.) frz. coute prov. colla ital. coltra sic. cutra tar. cultra. Prov. Mask. coisser.

Neben anate $(m)$ bezw. anite $(m)$ steht anítre( $m)$ com. nedra ital. anitra romg. anádra, doch ist überall die Accentverschiebung und der Antritt des $r$ jung. Ähnlich die von C. Michaelis Stud. S. 25 I Anm. angeführten Formen von árbutum $+r$.

Ich habe noch andere Tonversetzungen zu verzeichnen. 1. Vorrücken: Suffix eólus, vgl. darüber Mirisch Suffix -olus S. 27 ff.; $p a-$ riéte $(m)$ besser paréte $(m)$ : zuerst verschmolzen im Nom. ie zu $\rho$, dann verschwand das $i$ auch in den cass. obll. ebenso a a bẹte. In muliere $(m)$ hatte $i$ an $l$ einen festern Halt, doch wurde der Diphthong $i e$ in ie verwandelt, ebenso im ital. piéta: man mag daraus ein weiteres, doch nicht sehr schwer wiegendes Argument dafür ziehen, dafs ie einst ie gesprochen wurde. Ähnlich sind span. $y \delta$ Dios. Auf Suffixvertauschung beruhen cedrina u. a. Diez I $502 \mathrm{f}$., dazu bućina frz. buisine, ebenso frz. souris: sorex und berbex beeinflussen sich gegenseitig; sórïcem - berbẹcēm zu soricem berbícem; dagegen unterliegt berbęcem im ital. berbice ganz dem Einflusse der übrigen auf $e x$-icis; cervix, was ebenfalls auf berbex eingewirkt haben kann, bleibt der alten Form treu. ${ }^{2}$ Häufig in gelehrten Wörtern vgl. Diez I 503, man kann hinzufügen span. enté co hecticus.

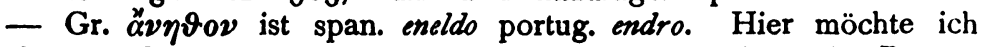
eher lat. Betonung anéthum annehmen, im Span. ist $l$, im Portug. $r$ eingeschoben; aus anétum entstand in letzterer Sprache aedro aédro ẽdro. Im Nprov. wird der Accent stets auf die vorletzte Silbe vorgeschoben, einige Beispiele sind oben gegeben, andere s. Duraud R. d. l. R. XXIV 159 ff., wo freilich nicht alles tadellos ist. - Die Fälle im Präsens der Verba mit tonloser Paenultima bedürfen weiter keines Kommentars.

Rückziehung: In érgere porgere cogliere coudre u. s. w. will Förster, Rhein. Mus. XXXIII 295 den alat. Accent érigere u. s. w.

1 C. Michaelis Stud. 242a, 299b leitet trueno von tonitrus ab; das Wort gehört $z \mathbf{u}$ tonus mit epenthetischem $r$ (etwas unter Einflufs von tronus = Himmel).

- Diez Gr. I 504 betont varice, was ebenfalls hierher gehört, wenn es richtig ist; andere geben virice an. 
sehen, allein mit Unrecht: das Accentgesetz ist aussnahmlos, wie jedes andere Lautgesetz, es ergreift alle Fälle. „Mehrere Verba der zweiten romanischen Konjugation wenden durch irrige Analogie den Accent des Präsens auf den Infinitif an", sagt Diez I 502 kurz und deutlich. - Eine zweite Klasse bilden die Ortsnamen; vgl. Ascoli, Arch. IV 46 I, Diez I 506. Aufser den dort genannten noch Lípanto Métaro, Trẹto in Gallien Nîmes Némausum u. a. Das letztgenannte fällt nicht in Betracht; im Altkeltischen ruhte der Hochton auf der ersten Silbe, die Römer nahmen den Namen zugleich mit seiner Betonung auf, das in unsern Schulen gebräuchliche Nemaísum ist weder keltisch noch lateinisch noch romanisch. Ähnlich verhält es sich mit Pésaro Métaro. Bei den übrigen mag sich fragen, ob sich die Neigung geltend macht, die Ortsnamen überhaupt auf der ersten Silbe zu betonen (eine Neigung, die früher oder später eingetreten sein kann) oder ob sich ein Rest der bei den Italisten gebräuchlichẻn Betonung bewahrt hat. Die von Diez I 505 für O'tranto gegebene Erklärung könnte nur für Namen fremder Gegenden, z. B. Lépanto passen. Brindisi (der Accent wird sogleich besprochen) zeigt in Übereinstimmung mit der griechischen im Gegensatz zur römischen Überlieferung heute eine richtigere Namens-

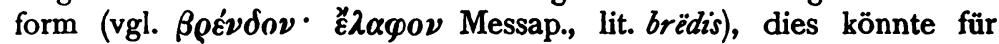
dic zweite Möglichkeit sprechen. Es wäre wünschenswert, dals jemand mit den gehörigen Hülfsmitteln versehen sämtliche süditalienischen Ortsnamen daraufhin untersuchte. - Die Zehnerzahlen: Gröber, Zeitschr. IV 3 I 2 setzt vźginti tréginta für alle Sprachen, quadrăginta u. s. w. fürs Prov. Frz. Ital. an. Setzt man sich über den Ausfall des $g$ vor $i$ nach dem Tone im Ital. Frz. Portug. hinweg, so ist kein Grund vorhanden, für Span. Portug. von quadrá $[g]$ inta abzugehen, ai wird $e i$ e. Es läge also Rückziehung des Accents auf den, den unterscheidenden Begriff enthaltenden Teil des zusammengesetzten Wortes vor. Allein weshalb betonte man dann nicht die erste Silbe? Darauf, dals $n$ im Nexus $n t$ keine Position bilde (Corssen II 2 I3, 66r u. a.) wird sich niemand berufen wollen. - trí folium Gr. I 507; im Gegensatz zu den Neubildungen "auf -ium wurde auch aus einen alten Worte ein (falsches) Primitivum auf $-u m$ gebildet: *trîf folum. Noch merkwürdiger ist aquifolium span. acebo portug. asevinho, aus aquifolium entstand span. * acebojo, -ojo hielt man für das Suffix ojo (Diez. II 326) und bildete ein Primitiv acebo. - Wenn in einigen ital. Wörtern auf zcius Gr. I 503 nebst sen. papecia Caix, Studi 435 Zurückziehung des Accents stattgefunden hat, so geht dies aus einem Streben icizus (2 silbig) an icem anzugleichen hervor. Mehrere der Gr. I 503 f. angeführten Beispiele sind gelehrte Wörter, von einigen anderen sprach ich in Verlaufe der Abhandlung, für manche, wie fícatum, dadiva u. a. weifs ich den Grund nicht. Über die Betonung von tabanus im Lat. wissen wir nichts, die Wörterbücher bezeichnen ohne hinreichenden Grund das zweite $a$ als lang; mlat. tavenus (Diez, Wb. 313) spricht eher für Kürze, ebenso span. tábano, die übrigen rom. Formen für 
Länge; entsprechende Wörter anderer indogermanischer Sprachen kenne ich nicht, die bei Diez verzeichnete Erklärung Ascolis ist lautlich unmöglich. An sich ist es wahrscheinlicher, dafs tábrnnus unter Einflufs der übrigen Wörter auf -ānus zu tabánus umgeändert wurde als umgekehrt tabănus zu tábannus. Miercoles = mércuri, wie Burda, Rev. p. fil. I 2 gesehen hat, ebenso Brindisi $=$ Brindisis.

Die Accentversetzungen in griechischen Wörtern sind mit einer grofsen Zahl schwieriger Fragen verbunden, so dafs ich hier ganz davon absehe; einige Beispiele, die mir gerade beachtenswert schienen, habe ich schon gebracht, sonst vgl. Diez, Gr. I 504. [Vgl. jetzt D' Ovidio oben S. $82 \mathrm{ff}$, dem ich meistens beistimme.]

Überblicken wir nun das ganze, so sehen wir, dafs, abgesehen von einigen sehr alten Fällen (vgl. S. 209), jede Sprache in der Synkope ihre eignen Wege geht, dafs auch da, wo mehrere Sprachen übereinstimmen in der Tilgung, dieselbe doch nicht überall gleichzeitig eingetreten ist. Sie ist aber überall alt: meist älter als unsere litterarischen Denkmäler, auch älter als die Entwickelung der specifischen Eigentümlichkeiten einzelner Gruppen: frz. rät. Übergang von $a$ in $e$, portug. Ausfall von $\underline{v} n \underline{v}$ (im Anlaut der Nachtonsilbe, nicht im Auslaut), aber jünger als das gallische Auslautgesetz. Sie unterbleibt eher, wenn die anlautenden und auslautenden Konsonanten nicht leicht verträglich sind mit einander (daher namentlich bei anlautender mehrfacher Konsonanz, bei auslautend $c^{\prime}$ ), tritt um so lieber ein, wenn beliebte Konsonantengruppen entstehen, bei anlautend $l$ (gemeinrom. * colpum polpum u. a.) $r s$ (weniger leicht $s c$ ) $n$ +ausl. Verschlufslaut. Im Verhältnis zur Lautabstufung ergiebt sich folgendes: nur im Frz. und zum Teil im Prov. ist diese letztere jünger, im Span. Portug. Rät. und dem gröfsten Teile des prov. jene. Die Sache erkläre ich mir so: die erste wichtigste Änderung, die das Lateinische im Munde der Kelten empfing, war die Verdrängung des lat. chromatisch-expiratorischen Accents durch den kelt. expiratorischen (vgl. Schuchardt, Zeitschr. IV 142), infolge dessen mufste der unmittelbar dem Accente folgende Vokal fallen $\_$- wurde $\mathrm{zu} \perp ; \perp \ldots \mathrm{zu} \perp \perp$. In einer folgenden Periode und im Span. Portug. Prov. von jeher verteilte sich die Macht des Accentes auf die Vokale, belastete dabei diese so sehr, dafs die Konsonanten darunter litten, und sich ihnen möglichst anglichen: tonlose werden tönend, tönende fallen. Wenn ich also nicht zögere, mit Schuchardt und Ascoli die Sondergestaltung des französischen Vokalismus keltischem Einflusse zuzuschreiben, so sehe ich dagegen in der Entwickelung des Konsonantismus eher ein paralleles Vorgehen beider Sprachzweige: es wäre ja freilich möglich, dafs während der Romanisierung, die ja sehr lange Zeit in Anspruch nahm, jener Wechsel im Wesen des Accents sich im Keltischen selbst vollzogen und aufs Romanische eingewirkt hätte. Beweisen lärst sich das mit unsern heutigen Hülfsmitteln noch nicht. 
Jenes, zu Anfang der Untersuchung dargelegte lateinische Gesetz sehen wir zum Teil im Romanischen fortwirken, monichus, umgekehrt pampanus u. a. begegneten auf verschiedenen Gebieten; aber wir dürfen diese Formen nicht als vulgärlat. bezeichnen, wenn wir nicht sogleich hinzufügen, dafs sie nur dialektisch, d. h. nur auf bestimmte Gegenden beschränkt sind. - Auch darin, dafs in der Verbindung $. l i+$ Kons. der Vokal am ehesten fält, setzt sich nur eine alte Neigung fort; vgl. balneum aus $\beta \alpha \lambda \alpha \nu \varepsilon \tau o \nu$, ulna aus olina (gr. o’hévy got. aleina) malva aus malaghva (gr. $\mu \alpha \lambda \alpha ́ \alpha \eta$ alb. muláge, die von Fick angesetzte Grundform malva ist falsch).

Ein besonderer Fall muls noch besprochen werden. Ein tonloser Vokal, der zwischen denselben Konsonanten eingeschlossen ist, wird gerne unterdrückt. Man wirft dies gewöhnlich mit dem Ausfall einer Silbe zur Dissimilation zusammen, obschon die beiden Erscheinungen ihrem Wesen und ihrem Resultate nach durchaus verschieden sind; im erstern Falle handelt es sich um einen physiologischen, im letztern um einen psychologischen Vorgang. Wenn ich átata spreche, so kann ich, da fast unmittelbar nach Lösung des Verschlusses an derselben Artikulationsstelle ein neuer Verschlufs gebildet werden soll, den zwischenliegenden Vokal ganz kurz, schwa-artig aussprechen; zuerst noch mit Offnung der Stimmritze, dann_ohne dieselbe $a t^{\prime \prime} t a$, schliefslich die zwei Konsonanten in einen áta. Ich kann aber auch, nachdem ich das erste ta gesprochen habe, dieses für das zweite halten, und also áta sprechen. Das erste wäre $a t(a) t a$, das zweite at(at)a. Auf letztern Wege erklären sich C: Michaelis und Caix ein vulgl. matinus, was gar nie existiert hat; ital. maltino span. matin frz. matin u. s. w. (auch das bekannte maitino) fordern mit Notwendigkeit (wie übrigens Ascoli längst im Arch. I an verschiedenen Stellen lehrt) ein Etymon mit $-t t-$, das auf dem eben beschriebenen Wege entstanden ist. So haben wir nun nach dem Tone: netto u. s.w.; ferner ital. pancia, da die Verschlufsbildung für $c^{\prime}$ ja dieselbe ist wie für $i$, und einige andere im Laufe der Abhandlung angeführte Beispiele.

Was sich weiter etwa in den Einzelsprachen zur Geschichte der Lautentwickelungen ergeben hat, habe ich jeweilen mit der Kürze angedeutet, die ein Zeitschriftenaufsatz bedingt, der doch nur Bahnen ebnen, Materialien vorläufig disponieren, nicht ein ganzes Gebäude auf bauen soll.

Mit ein paar Worten möchte ich noch auf rdus zurückkommen. Diez zweifelt an der Möglichkeit von Neubildungen Gr. II 321 , doch habe ich aus verschiedenen Dialokten solche angeführt, und es giebt deren noch mehr; doch zeigt namentlich der Mangel eines festen Anwendungsprincips, wie schwach die Lebensfähigkeit ist, im Lat. sind sie ursprünglich von neutralen Stämmen abgebildet: frigidus = frígesdos; lucidus loukesdos (nicht von lüc-), im Rom. aber auch von Adj., vgl. namentlich ital. spurcido, orbido. -idus wird nicht selten mit dem häufigern zcus vertauscht, zunächst bei Adj.

Zoiteohr. f. rom. Phll. VIII. 
auf cidus und gidus, wo also Metathesis eintritt sard. pidigu, mail. ledeg, piem. reidi u. a. oben verzeichnete; aufserdem sard. luzzigu, tevec in Fassa (Arch. I 350) rancico tosk. (Fanf.) agord. logod., vgl. Mussafia, B. 116 Anm. 2; berg. lambec ib., vgl. span. enjalbeguar; dann auch trespec Mussafia B. I16; häufig geht $d$ in $l$ über neap. tiepolo, ebenso bei Rusio, friul. fumul Arch. II 408 aven. cospolo Mussafia, B. 47 ; trespolo Arch. II 408, wo Ascolis Bemerkungen zu vergleichen sind.

W. Meyer.

Nachtrag. Ich hatte den Artikel tonto Wb. II $\mathrm{S} .492$ übersehen. Es ist klar, dafs tönitus im Span. nur tuendo geben kann. Ital. portug. tọnto span. tonto weisen auf tōntus bezw. tuntus zurück. $\mathrm{Zu}$ tundere stofsen konnte neben tusum tunsum ein Praet. Iunditum tuntum gebildet werden wie zu pandere neben passum pansum panditum, die Bedeutung ist „abgestumpft“. - Nach den obigen Ausführungen fällt branca = biramica, was Thurneysen Keltorom. s. v.

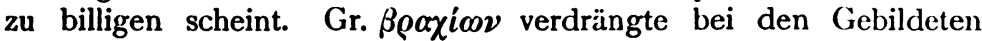
ein wahrscheinlich ähnliches klingendes lat. Wort, eben dieses im Volksmunde fortlebende branca. Die Wurzel ist bhrenc, dieselbe, die wir im deutschen „bringen“ haben. Im Griech. verlor sich wie in vielen andern Fällen die Aspiration vor $e, k$ wurde nach $n \mathrm{zu}$ $\chi$, in Tieftonsilbe erhalten wir $\beta \varrho \alpha \chi-;$ im Lat. entspricht tieftonigen rn an wie z. B. in trans, also grof. brncía oder $b \bar{r} n c \dot{a}$. 